\title{
Near-ideal molecule-based Haldane spin chain
}

\author{
Robert C. Williams, ${ }^{1}$ William J. A. Blackmore, ${ }^{1}$ Samuel P. M. Curley, ${ }^{1}$ Martin R. Lees, ${ }^{1}$ Serena M. Birnbaum, ${ }^{2}$ \\ John Singleton, ${ }^{2}$ Benjamin M. Huddart, ${ }^{3}$ Thomas J. Hicken, ${ }^{3}$ Tom Lancaster, ${ }^{3}$ Stephen J. Blundell, ${ }^{4}$ Fan Xiao, ${ }^{5,6}$ \\ Andrew Ozarowski, ${ }^{7}$ Francis L. Pratt, ${ }^{8}$ David J. Voneshen, ${ }^{8}$ Zurab Guguchia, ${ }^{9}$ Christopher Baines, ${ }^{9}$ John A. Schlueter, ${ }^{10,11}$ \\ Danielle Y. Villa, ${ }^{12}$ Jamie L. Manson, ${ }^{12,}{ }^{*}$ and Paul A. Goddard ${ }^{1, \dagger}$ \\ ${ }^{1}$ Department of Physics, University of Warwick, Gibbet Hill Road, Coventry CV4 7AL, United Kingdom \\ ${ }^{2}$ National High Magnetic Field Laboratory, Los Alamos National Laboratory, Los Alamos, New Mexico 87545, USA \\ ${ }^{3}$ Department of Physics, Durham University, South Road, Durham DH1 3LE, United Kingdom \\ ${ }^{4}$ Department of Physics, Clarendon Laboratory, Oxford University, Parks Road, Oxford OX1 3PU, United Kingdom \\ ${ }^{5}$ Department of Chemistry and Biochemistry, University of Bern, Freiestrasse 3, CH-3012 Bern, Switzerland \\ ${ }^{6}$ Laboratory for Neutron Scattering and Imaging, Paul Scherrer Institut, CH-5232 Villigen PSI, Switzerland \\ ${ }^{7}$ National High Magnetic Field Laboratory, Florida State University, Tallahassee, Florida 32310, USA \\ ${ }^{8}$ ISIS Facility, STFC Rutherford Appleton Laboratory, Chilton, Oxfordshire OX11 OQX, United Kingdom \\ ${ }^{9}$ Laboratory for Muon Spin Spectroscopy, Paul Scherrer Institut, CH-5232 Villigen PSI, Switzerland \\ ${ }^{10}$ Materials Science Division, Argonne National Laboratory, Argonne, Illinois 60439, USA \\ ${ }^{11}$ Division of Materials Research, National Science Foundation, 2415 Eisenhower Avenue, Alexandria, Virginia 22314, USA \\ ${ }^{12}$ Department of Chemistry and Biochemistry, Eastern Washington University, 226 Science, Cheney, Washington 99004, USA
}

(Received 30 August 2019; revised manuscript received 18 November 2019; published 27 January 2020)

\begin{abstract}
The molecular coordination complex $\mathrm{NiI}_{2}(3,5 \text {-lut })_{4}$ [where $(3,5$-lut $)=(3,5$-lutidine $\left.)=\left(\mathrm{C}_{7} \mathrm{H}_{9} \mathrm{~N}\right)\right]$ has been synthesized and characterized by several techniques including synchrotron x-ray diffraction, electron-spin resonance, superconducting quantum interference device magnetometry, pulsed-field magnetization, inelastic neutron scattering, and muon spin relaxation. Templated by the configuration of 3,5-lut ligands the molecules pack in-registry with the Ni-I $\cdots$ I-Ni chains aligned along the $c$ axis. This arrangement leads to an uncommon through-space I ... I magnetic coupling which is directly measured in this work. The net result is a near-ideal realization of the $S=1$ Haldane chain with $J=17.5 \mathrm{~K}$ and energy gaps of $\Delta^{\|}=5.3 \mathrm{~K} \Delta^{\perp}=7.7 \mathrm{~K}$, split by the easy-axis single-ion anisotropy $D=-1.2 \mathrm{~K}$. The ratio $D / J=-0.07$ affords one of the most isotropic Haldane systems yet discovered, while the ratio $\Delta_{0} / J=0.40(1)$ (where $\Delta_{0}$ is the average gap size) is close to its ideal theoretical value, suggesting a very high degree of magnetic isolation of the spin chains in this material. The Haldane gap is closed by orientation-dependent critical fields $\mu_{0} H_{\mathrm{c}}^{\|}=5.3 \mathrm{~T}$ and $\mu_{0} H_{\mathrm{c}}^{\perp}=4.3 \mathrm{~T}$, which are readily accessible experimentally and permit investigations across the entirety of the Haldane phase, with the fully polarized state occurring at $\mu_{0} H_{\mathrm{s}}^{\|}=46.0 \mathrm{~T}$ and $\mu_{0} H_{\mathrm{s}}^{\perp}=50.7 \mathrm{~T}$. The results are explicable within the so-called fermion model, in contrast to other reported easy-axis Haldane systems. Zero-field magnetic order is absent down to $20 \mathrm{mK}$ and emergent end-chain effects are observed in the gapped state, as evidenced by detailed low-temperature measurements.
\end{abstract}

DOI: 10.1103/PhysRevResearch.2.013082

\section{INTRODUCTION}

Background. Molecule-based magnets have proved a highly successful avenue in achieving desired lowdimensional geometries [1-3]. Several classes of materials which host quantum-disordered nonmagnetic ground states can undergo magnetic field and pressure driven quantum phase transitions (QPTs), where the magnetic ground states

\footnotetext{
*jmanson@ewu.edu

†p.goddard@warwick.ac.uk
}

Published by the American Physical Society under the terms of the Creative Commons Attribution 4.0 International license. Further distribution of this work must maintain attribution to the author(s) and the published article's title, journal citation, and DOI. undergo a dramatic reorganization and physical properties exhibit characteristic behavior [4,5].

An important asset of molecule-based systems is the modest energy scale of their exchange interaction strengths, which makes their QPTs experimentally accessible, thus providing a vital opportunity to test the predictions of theory and simulations. Invaluable insight has been afforded by the intensive experimental study of prototypical realizations of models including: the ideal $S=1 / 2$ chain material $\mathrm{Cu}\left(\mathrm{C}_{4} \mathrm{H}_{4} \mathrm{~N}_{2}\right)\left(\mathrm{NO}_{3}\right)_{2}$ [6]; the strong-leg and strong-rung two-leg spin-ladder systems $\left(\mathrm{C}_{7} \mathrm{H}_{10} \mathrm{~N}\right)_{2} \mathrm{CuBr}_{4}$ (DIMPY) [7] and $\left(\mathrm{C}_{5} \mathrm{H}_{12} \mathrm{~N}\right)_{2} \mathrm{CuBr}_{4}$ (BPCB) [8], respectively; the $S=1 / 2$ dimer compound $[\mathrm{Cu}($ pyrazine $)($ glycinate $)]\left(\mathrm{ClO}_{4}\right)$ [9]; the large- $D$ system $\mathrm{NiCl}_{2} 4 \mathrm{SC}\left(\mathrm{NH}_{2}\right)_{2}$ (DTN) [10]; and the spinliquid compound $\left(\mathrm{C}_{4} \mathrm{H}_{12} \mathrm{~N}_{2}\right) \mathrm{Cu}_{2} \mathrm{Cl}_{6}$ (PHCC) [11]. Of note is that several of these halide containing materials rely on through-space magnetic couplings, i.e., two-halide exchange, 
rather than that offered by bridging ligands. Here we present a material of this kind supported by highly unusual noncovalent I ... I interactions.

Pioneering work by Haldane demonstrated that Heisenberg antiferromagnetic (AFM) chains of integer spins host a disordered ground state which is topologically distinct from the well-known, spin-half analog (the Tomonaga-Luttinger liquid) $[12,13]$. The significance of this was recognized via the award of the 2016 Nobel Prize in Physics. The Haldane phase is protected by its eponymous energy gap, above which excitations propagate. Fractional excitations manifesting as $S=1 / 2$ degrees of freedom exist at the end chains, due to the symmetry-protected bulk topological phase. This phenomenon arises naturally out of the valence bond solid model [14], and has been experimentally observed in several Haldane compounds [15-17]. Higher concentrations of $S=1 / 2$ edgestate excitations may be induced by breaking the chains via the introduction of nonmagnetic sites, which has been shown to result in magnetic ordering in $\mathrm{PbNi}_{2} \mathrm{~V}_{2} \mathrm{O}_{8}$ due to the almost critical interchain exchange coupling $[18,19]$.

It has been demonstrated that extending the Hamiltonian of the AFM $S=1$ chain to include single-ion anisotropy (SIA) and interchain interactions gives rise to a rich phase diagram [20-22]. The resultant Hamiltonian is given by

$$
\begin{aligned}
\hat{\mathcal{H}}= & D \sum_{i}\left(\hat{\boldsymbol{S}}_{i}^{z}\right)^{2}+J \sum_{\langle i, j\rangle} \hat{\boldsymbol{S}}_{i} \cdot \hat{\boldsymbol{S}}_{j}+J_{\perp} \sum_{\left\langle i, j^{\prime}\right\rangle} \hat{\boldsymbol{S}}_{i} \cdot \hat{\boldsymbol{S}}_{j^{\prime}} \\
& +\mu_{\mathrm{B}} \mu_{0} \sum_{i} \boldsymbol{H} \cdot \boldsymbol{g} \cdot \hat{\boldsymbol{S}}_{i},
\end{aligned}
$$

where $J$ and $J_{\perp}$ are the dominant intrachain and weaker interchain exchange interactions between $S=1$ moments, respectively. Here, angular brackets denote sums taken over unique pairs of nearest-neighbor spins, primed indices refer to spins on adjacent chains, $D$ is the axial SIA parameter, and the final term is the usual Zeeman interaction for an external magnetic field $\boldsymbol{H}$. The ensuing phase diagram has recently been extended to consider the rhombic SIA parameter $E$ [23].

In order to exploit the interplay between crystal structure and magnetic behavior in $S=1$ systems, and ultimately tune and control the parameters $J$ and $D$, we have synthesized and characterized a series of $\mathrm{Ni}$ (II)-containing materials. Different bridging and nonbridging ligands enable the exploration of low-dimensional phase diagrams, resulting in dramatically different magnetic behavior [24-26]. In this work we report the discovery and characterization of a $S=1$ molecule-based AFM chain $\mathrm{NiI}_{2}(3,5 \text {-lut })_{4}$ where $(3,5$-lut) $=(3,5$-lutidine $)=\left(\mathrm{C}_{7} \mathrm{H}_{9} \mathrm{~N}\right)$. Magnetometry measurements reveal the AFM intrachain coupling $J=17.5(2) \mathrm{K}$, which together with the axial SIA parameter $D=-1.2(1) \mathrm{K}$ results in two triplet energy gaps $\Delta^{\|}=0.46(1) \mathrm{meV}=5.3(1) \mathrm{K}$ and $\Delta^{\perp}=0.66(1) \mathrm{meV}=7.7(1) \mathrm{K}$, as observed via inelastic neutron scattering (INS). The Haldane gap may be closed by the anisotropic critical magnetic fields $\mu_{0} H_{\mathrm{c}}^{\|}=5.3(1) \mathrm{T}$ and $\mu_{0} H_{\mathrm{c}}^{\perp}=4.3(1) \mathrm{T}$, and the system is saturated at $\mu_{0} H_{\mathrm{s}}^{\|}=$ 46.0(4) $\mathrm{T}$ and $\mu_{0} H_{\mathrm{s}}^{\perp}=50.7(8) \mathrm{T}$. The ratio $D / J=-0.07(1)$ makes this the among the most isotropic Haldane chains reported to date, and the modest energy scales lead to experimentally accessible critical fields, enabling the use of superconducting magnets found at beamline facilities to explore the entire Haldane phase. To this end, we performed a transverse-field muon spin relaxation $\left(\mu^{+} \mathrm{SR}\right)$ study of the field-driven closure of the Haldane gap, while complementary zero-field $\mu^{+}$SR measurements confirm the absence of zerofield magnetic ordering down to $T=20 \mathrm{mK}$.

For the isotropic Haldane phase with $D=0$, analytical and numerical approaches postulate two- and three-particle continua in the zero-field magnetic excitation spectra at the Brillouin-zone boundary $(k=\pi)$ and center $(k=0)$, respectively (see Ref. [27] and references therein). These continua augment the well-established lowest-lying "single-magnon" mode defining the Haldane gap $\Delta$. However, their existence has not yet been demonstrated experimentally [28-30]. A nearly isotropic Haldane chain material such as $\mathrm{NiI}_{2}(3,5-l u t)_{4}$ is therefore highly desirable to help verify this prediction. $\mathrm{NiI}_{2}(3,5-\mathrm{lut})_{4}$ also affords the possibility for controlled introduction of both bond and site disorder, which makes this compound a model system for investigations into the physics of the Haldane phase.

Single-ion anisotropy in the Haldane phase. In zero field, the presence of axial SIA serves to lift the degeneracy of the excited triplet states above the Haldane ground state, resulting in energy levels given by [31]

$$
\Delta^{\|}=\Delta_{0}+1.41 D, \quad \Delta^{\perp}=\Delta_{0}-0.57 D,
$$

for the singlet longitudinally polarized excitation mode (denoted $\|$, corresponding to the $S_{z}=0$ triplet state) and the transverse-polarized doublet $\left(\perp\right.$, corresponding to the $S_{z}=$ \pm 1 triplet states). Longitudinal and transverse is defined with respect to the unique $z$ direction, which here coincides with the chain axis $c$. The intrinsic Haldane gap $\Delta_{0}=0.41 \mathrm{~J}$ depends solely on the intrachain exchange coupling [32-36]. This energy-level arrangement may be compared to the singleion equivalent for isolated $S=1$ moments subject to axial SIA, where the effective SIA parameter $D_{\text {eff }}$ is given by

$$
D_{\text {eff }}=\Delta^{\perp}-\Delta^{\|}=-1.98 D \text {. }
$$

Note that $D_{\text {eff }}$ and $D$ have opposite signs.

There are several different quantum-field-theoretical models which describe the evolution of these energy levels under the application of magnetic fields. These models yield anisotropic values of the critical fields at which the Zeeman interaction drives a triplet state lower in energy than the Haldane ground state.

The field-dependent energy levels of the "fermion" (and equivalent "perturbative") model [37-40] map directly onto the isolated $S=1$ single-ion description [41], with the effective axial SIA term $D_{\text {eff }}$ given by Eq. (3). The fermion model predicts the following critical fields:

$$
g_{\|} \mu_{\mathrm{B}} \mu_{0} H_{\mathrm{c}}^{\|}=\Delta^{\perp}, \quad g_{\perp} \mu_{\mathrm{B}} \mu_{0} H_{\mathrm{c}}^{\perp}=\sqrt{\Delta^{\|} \Delta^{\perp}},
$$

for fields parallel and perpendicular to the unique axis.

The "boson" (and equivalent "macroscopic") model $[37,42]$ differs from the fermion model for fields perpendicular to the unique axis, particularly in the region close to the field-driven closure of the Haldane gap. The boson model predicts the following anisotropic critical fields:

$$
g_{\|} \mu_{\mathrm{B}} \mu_{0} H_{\mathrm{c}}^{\|}=\Delta^{\perp}, \quad g_{\perp} \mu_{\mathrm{B}} \mu_{0} H_{\mathrm{c}}^{\perp}=\Delta^{\|} .
$$




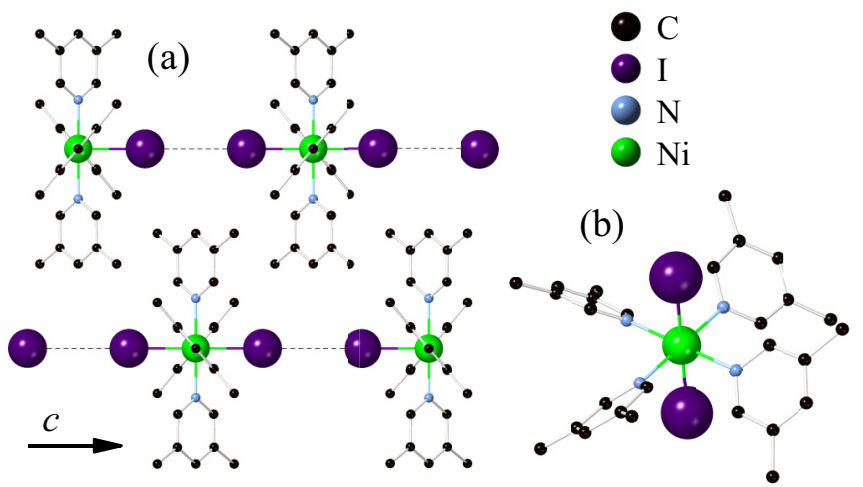

FIG. 1. (a) The chain structure of $\mathrm{NiI}_{2}(3,5 \text {-lut })_{4}$, viewed along the [110] direction, normal to the chain axis $c$ ( $\mathrm{H}$ atoms are omitted for clarity). (b) The local crystal environment of each $\mathrm{Ni}$ (II) ion.

Note that for the case of a negative SIA parameter $D<0$ (easy axis), then $\Delta^{\|}<\Delta^{\perp}$ and therefore both the fermion and boson models predict $H_{\mathrm{c}}^{\|}>H_{\mathrm{c}}^{\perp}$ (and vice versa for the easy-plane case).

The fermion model has been shown to consistently describe the behavior of the easy-plane compounds $\mathrm{Ni}\left(\mathrm{C}_{2} \mathrm{H}_{8} \mathrm{~N}_{2}\right)_{2} \mathrm{NO}_{2} \mathrm{ClO}_{4}$ (NENP) [43] and $\mathrm{Ni}\left(\mathrm{C}_{5} \mathrm{H}_{14} \mathrm{~N}_{2}\right)_{2} \mathrm{~N}_{3}\left(\mathrm{PF}_{6}\right)$ (NDMAP) [44], as it is able to simultaneously account for the anisotropic critical fields and the triplet energy levels. In contrast, the boson model has been shown to successfully describe the behavior of the two easy-axis Haldane compounds $\mathrm{PbNi}_{2} \mathrm{~V}_{2} \mathrm{O}_{8}$ and $\mathrm{SrNi}_{2} \mathrm{~V}_{2} \mathrm{O}_{8}$ [45-47]. It is believed that the success of the boson model for these compounds is due to their easy-axis anisotropy and critical interchain coupling interactions $\left(J_{\perp}\right)$, which place them close to the phase boundary between the Haldane and ordered Ising AFM phases.

\section{RESULTS}

Structural determination. Synchrotron single-crystal $x$-ray-diffraction measurements were performed to solve the crystal structure of $\mathrm{NiI}_{2}(3,5 \text {-lut })_{4}$ at $T=100(2) \mathrm{K}$. Details of the structural refinement can be found in the Appendix. $\mathrm{NiI}_{2}(3,5 \text {-lut })_{4}$ crystallizes in the space group $P 4 / n n c$, and comprises linear $\mathrm{Ni}-\mathrm{I} \cdot \ldots \mathrm{I}-\mathrm{Ni}$ chains running parallel to the $c$ axis, as shown in Fig. 1(a). The intrachain Ni-Ni separation is $c=9.9783(2) \AA$, where the magnetic superexchange interaction $J$ is mediated via the two iodine ions, and the linear configuration suggests AFM interactions according to the Goodenough-Kanamori rules [48-50].

The local crystal environment of each $\mathrm{Ni}$ ion is a tetragonally elongated $\mathrm{NiI}_{2} \mathrm{~N}_{4}$ octahedron, shown in Fig. 1(b), where the unique axial direction coincides with the chain axis $\boldsymbol{c}$. The $\mathrm{Ni}-\mathrm{N}$ and $\mathrm{Ni}-\mathrm{I}$ bond lengths are 2.123 and $2.833 \AA$, respectively. The chains running parallel to $c$ lie on the axes of fourfold rotational symmetry operators, which preclude the existence of rhombic SIA, and we therefore expect the magnetic behavior of $\mathrm{NiI}_{2}(3,5 \text {-lut })_{4}$ to be described by the Hamiltonian in Eq. (1).

The nonbridging lutidine ligands serve to separate adjacent chains within the $a b$ plane, which are offset by $c / 2$. The shortest $\mathrm{Ni}-\mathrm{Ni}$ distance is actually between ions on neighboring chains, however, there is no clear exchange pathway in this direction. The rings of the lutidine ligands are canted by $41.57^{\circ}$ with respect to the $a b$ plane, forming a propellerlike arrangement about the chain axes.

Not only do the methyl-substituents of the lutidine ligands in $\mathrm{NiI}_{2}(3,5 \text {-lut })_{4}$ create large interchain separations but they influence the crystal packing and prompt proper alignment of $\mathrm{Ni}-\mathrm{I} \cdots \mathrm{I}-\mathrm{Ni}$ chains. While $\mathrm{NiI}_{2}$ (pyridine) ) $_{4}$ is known [51], the molecules pack in such a way that potential I $\cdots$ I interactions are negated, making $\mathrm{NiI}_{2}(3,5 \text {-lut })_{4}$ particularly interesting. It is now possible to directly measure the magnetic exchange strength propagated by I $\cdots$ I couplings.

Magnetometry. Magnetic susceptibility measurements were performed on a powder sample of $\mathrm{NiI}_{2}(3,5 \text {-lut })_{4}$ using a superconducting quantum interference device (SQUID) magnetometer and the results are shown in Fig. 2(a). Upon cooling, a broad peak in the molar susceptibility $\chi_{\mathrm{m}}(T)$ is visible at around $20 \mathrm{~K}$ which indicates the buildup of short-range correlations and is typical for an AFM chain. As temperature is decreased further, a local minimum is reached at around $2 \mathrm{~K}$, due to the presence of additional paramagnetic spins. Importantly, there is no evidence in $\chi_{\mathrm{m}}(T)$ for a transition to magnetic long-range order (LRO) down to the lowest measured temperature. In fact, as will be discussed later, our muon spin-relaxation study demonstrates that in zero field $\mathrm{NiI}_{2}$ (3, 5-lut $)_{4}$ does not undergo magnetic ordering for temperatures down to $T=20 \mathrm{mK}$, indicating a small ratio $\left|J_{\perp}\right| / J$. Taken together with the small ratio $|D| / J$ revealed by the INS results, discussed below, this places $\mathrm{NiI}_{2}(3,5 \text {-lut })_{4}$ in the gapped Haldane region of the theoretically derived phase diagram $[20,21]$.

The susceptibility data are fitted to a model that describes an ideal isotropic Haldane chain $[52,53]$ as well as an additional term accounting for the presence of a small number of paramagnetic moments. Despite the isotropic approximation, this model is seen to account for the $\chi_{\mathrm{m}}(T)$ data extremely well across the entire measured temperature range $(1.8 \leqslant$ $T \leqslant 300 \mathrm{~K}$ ), as shown in Fig. 2(a). The extracted fit parameters yield estimates for the intrachain exchange interaction strength $J=18.27(4) \mathrm{K}$ and the average Haldane gap $\Delta_{0}=$ 7.26(1) K. The resultant magnitude of the Haldane gap with respect to the intrachain exchange strength $\Delta_{0} / J=0.397(1)$ is found to be close to the theoretically expected value of 0.41 for the ideal Haldane chain [32-36]. The fitted value of the powder-average $g$ factor is $g=2.197(1)$.

The presence of paramagnetic moments at low temperatures has been observed in susceptibility studies of related Haldane systems, for example NENP [17], and ascribed to the emergence of $S=1 / 2$ degrees of freedom at the chain ends within the Haldane phase, which arise due to lattice defects and crystallite boundaries within the powder sample. While we expect the presence of end-chain spins in our material at low temperatures (see the discussion of the electron-spinresonance data, below), we note that the $\chi_{\mathrm{m}}(T)$ data can be fit equally well with the addition of a small proportion $(\approx 1-3 \%)$ of either $S=1 / 2$ end chain moments or $S=1$ impurities.

Magnetization measurements were performed on a powder sample of $\mathrm{NiI}_{2}(3,5 \text {-lut })_{4}$ in quasistatic magnetic fields $\mu_{0} H \leqslant 7 \mathrm{~T}$, with results shown in Fig. 2(b). A clear kink 

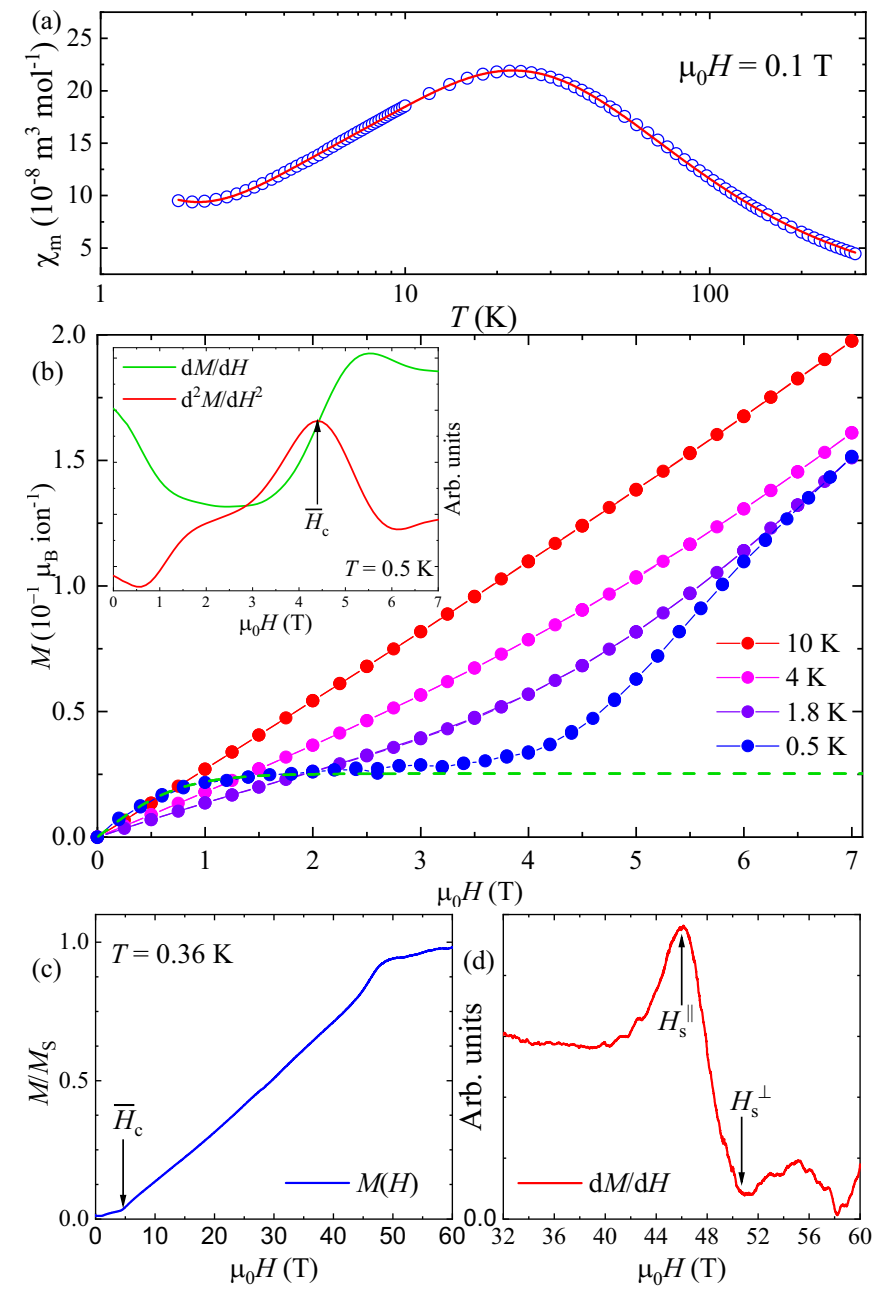

FIG. 2. Powder magnetometry results for $\mathrm{NiI}_{2}$ (3, 5-lut) . (a) Magnetic susceptibility data (circles). The solid line represents a fit to the data over the entire measured temperature range using the model described in the text. (b) Quasistatic magnetization data, where the lowest temperature $(T=0.5 \mathrm{~K})$ data have been fit to a paramagnetic model for the field range $\mu_{0} H \leqslant 2 \mathrm{~T}$ (dashed green line). Inset: The first and second derivatives of the low-temperature $M(H)$ data, with respect to magnetic field. (c) Pulsed-field magnetization data and (d) their derivative, measured at $T=0.36 \mathrm{~K}$.

is visible in the data close to $4 \mathrm{~T}$, which becomes more pronounced in lower temperature data sets. We ascribe this kink to the field-driven closure of the Haldane gap, as the Zeeman interaction drives the energy of a triplet excited state below that of the Haldane ground state [54]. The powderaverage value of the critical field $\mu_{0} \bar{H}_{\mathrm{c}}=4.4(2) \mathrm{T}$ is obtained by locating the peak in $d^{2} M / d H^{2}$ at $T=0.5 \mathrm{~K}$, shown in the inset to Fig. 2(b), corresponding to the kink in the $M(H)$ magnetization data.

Further evidence for the presence of the magnetically disordered Haldane phase for fields below $H_{\mathrm{c}}$ comes from the suppression of magnetization in this region as temperature is decreased from $T=10 \mathrm{~K}$ [Fig. 2(b)]. A magnetic response is still visible for low magnetic fields $\mu_{0} H<2 \mathrm{~T}$ for $M(H)$ data measured at $T=0.5 \mathrm{~K}$ in the iQuantum ${ }^{3} \mathrm{He}$ insert of

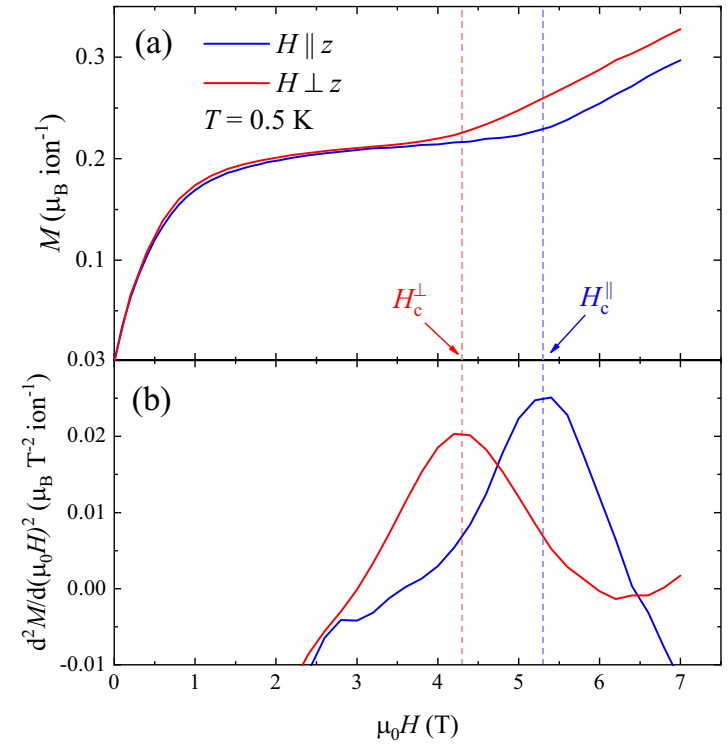

FIG. 3. (a) Single-crystal magnetization measurements performed with magnetic fields parallel $(\|)$ and perpendicular $(\perp)$ to the unique local axis in $\mathrm{NiI}_{2}(3,5 \text {-lut })_{4}$, which coincides with the chain direction. (b) The second derivative of magnetization with respect to field yields the anisotropic critical fields.

the SQUID magnetometer. As can be seen from the successful fit of the low-field data to an $S=1 / 2$ Brillouin function (dashed green line), this low-field response resembles that of a collection of free paramagnetic spins. The paramagnetic contribution is attributed to a combination of the background of the iQuantum insert, as well as the presence of $S=1 / 2$ degrees of freedom at the chain ends within the Haldane phase (and the possibility of a small number of $S=1$ impurities). It has proved difficult to reliably subtract the background contribution from the sample signal, which becomes very small in the gapped phase. We are therefore not able to isolate the intrinsic sample response to the low-temperature, lowfield magnetometry. We note, however, that the background magnetization is field independent for $\mu_{0} H>1.5 \mathrm{~T}$ and becomes significantly smaller than the sample response as the Haldane gap starts to close above $4 \mathrm{~T}$. A full discussion of the background arising from the iQuantum insert is included in the Appendix.

In order to investigate the SIA-induced orientation dependence of the critical magnetic fields, low-temperature $(T=$ $0.5 \mathrm{~K}$ ) magnetization measurements were made on a small single crystal of $\mathrm{NiI}_{2}(3,5 \text {-lut })_{4}$ with magnetic fields oriented parallel $(\|)$ and perpendicular $(\perp)$ to the unique $c$ axis, with results shown in Fig. 3. Again, an isotropic paramagnetic response is visible at low fields, consistent with the combination of background plus chain ends; however, the subsequent upturn in $M(H)$ is clearly seen to differ for the two magnetic field orientations. By examining the second derivative $d^{2} M / d H^{2}$ [Fig. 3(b)], the critical field values $\mu_{0} H_{\mathrm{c}}^{\|}=5.3(1) \mathrm{T}$ and $\mu_{0} H_{\mathrm{c}}^{\perp}=4.3(1) \mathrm{T}$ are determined. The observation that $H_{\mathrm{c}}^{\|}>$ $H_{\mathrm{c}}^{\perp}$ constitutes unambiguous evidence for nonzero easy-axis SIA in this compound.

Pulsed-field magnetization measurements were performed on a powder sample of $\mathrm{NiI}_{2}(3,5 \text {-lut })_{4}$, with representative 
results shown in Fig. 2(c). As for the measurements under quasistatic fields, a kink is clearly visible where the Haldane gap is closed at $\bar{H}_{\mathrm{c}}$. As the magnetic field is increased further, the magnetization response increases approximately linearly until close to the saturation field, where the curvature is typical of a one-dimensional system [55]. The peak in $d^{2} M / d H^{2}$ is used to determine the value of the critical field $\mu_{0} \bar{H}_{\mathrm{c}}=$ 4.3(3) T, which agrees closely with the value deduced from the SQUID magnetometry measurements. Using the result that $D<0$ in this system then, following the mean-field calculations in Ref. [56], the anisotropic saturation fields are found to be

$$
\begin{aligned}
g_{\|} \mu_{\mathrm{B}} \mu_{0} H_{\mathrm{s}}^{\|} & =2(2 J-|D|), \\
g_{\perp} \mu_{\mathrm{B}} \mu_{0} H_{\mathrm{s}}^{\perp} & =2(2 J+|D|),
\end{aligned}
$$

where it has been assumed that $\left|J_{\perp}\right| \ll J$. As shown in Fig. $2(\mathrm{~d}), \mu_{0} H_{\mathrm{s}}^{\|}=46.0(4) \mathrm{T}$ and $\mu_{0} H_{\mathrm{s}}^{\perp}=50.7(8) \mathrm{T}$ are readily identifiable as the beginning and end, respectively, of the decrease in $d M / d H$ accompanying the plateau in $M(H)$ at saturation. Taking the anisotropic $g$ factors $g_{\|, \perp}=$ 2.13(1), 2.19(1) from the electron-spin-resonance (ESR) results discussed below, Eq. (6) yield values for the SIA parameter $D=-1.2(3) \mathrm{K}$ and intrachain exchange coupling $J=17.5(2) \mathrm{K}$, which is in reasonable agreement with the estimate obtained from the fit to the susceptibility data.

Inelastic neutron scattering. In order to examine the magnetic excitations of $\mathrm{NiI}_{2}(3,5-\mathrm{lut})_{4}$ and resolve the SIA-split Haldane energy gaps in zero field, INS measurements were performed on a powder sample using the LET instrument at ISIS, UK. Representative data collected at $T=1.8 \mathrm{~K}$ for incident neutron energy $E_{\mathrm{i}}=2.2 \mathrm{meV}$ are shown as a $(|Q|, E)$ spectrum in Fig. 4(a), where $|Q|$ and $E$ denote momentum and energy transfers, respectively. Corresponding $T=12 \mathrm{~K}$ data (shown in the Appendix) are treated as a background, and have been subtracted from the data. The two components of the triplet excitations are visible as bands of excitations for energies above $E \approx 0.4$ and $0.6 \mathrm{meV}$. Significant intensity is recorded for energy transfers below $E \approx 0.2 \mathrm{meV}$ at all temperatures measured and is caused in part by the elastic and quasielastic contribution from nuclear scattering. However, after subtraction some low-energy intensity still remains and grows with decreasing temperature. This could have a magnetic origin, most likely the buildup of correlations between the end chain moments.

The Haldane gaps may be clearly quantified by inspecting an energy cut through the data: Figure 4(b) shows cuts obtained by integrating over the full measured range of $|Q|$ for the background-subtracted data for $T=1.8,3$, and $7 \mathrm{~K}$ and $E_{i}=2.2 \mathrm{meV}$. The SIA-split Haldane gaps are visible as the low-energy onsets of the bands of spin excitations, where the large density of states at each excitation band minimum leads to a rapid increase in scattering intensity at energies centered around each gap energy $[57,58]$. The excitation bands become more pronounced as temperature is lowered, confirming that these features originate in the magnetic contribution to the scattering. The line shapes of the two features vary a little for data collected at additional values of $E_{i}$ (see the Appendix), but their relative size remains similar to the data shown in Fig. 4(b), i.e., the higher energy feature is larger than that of
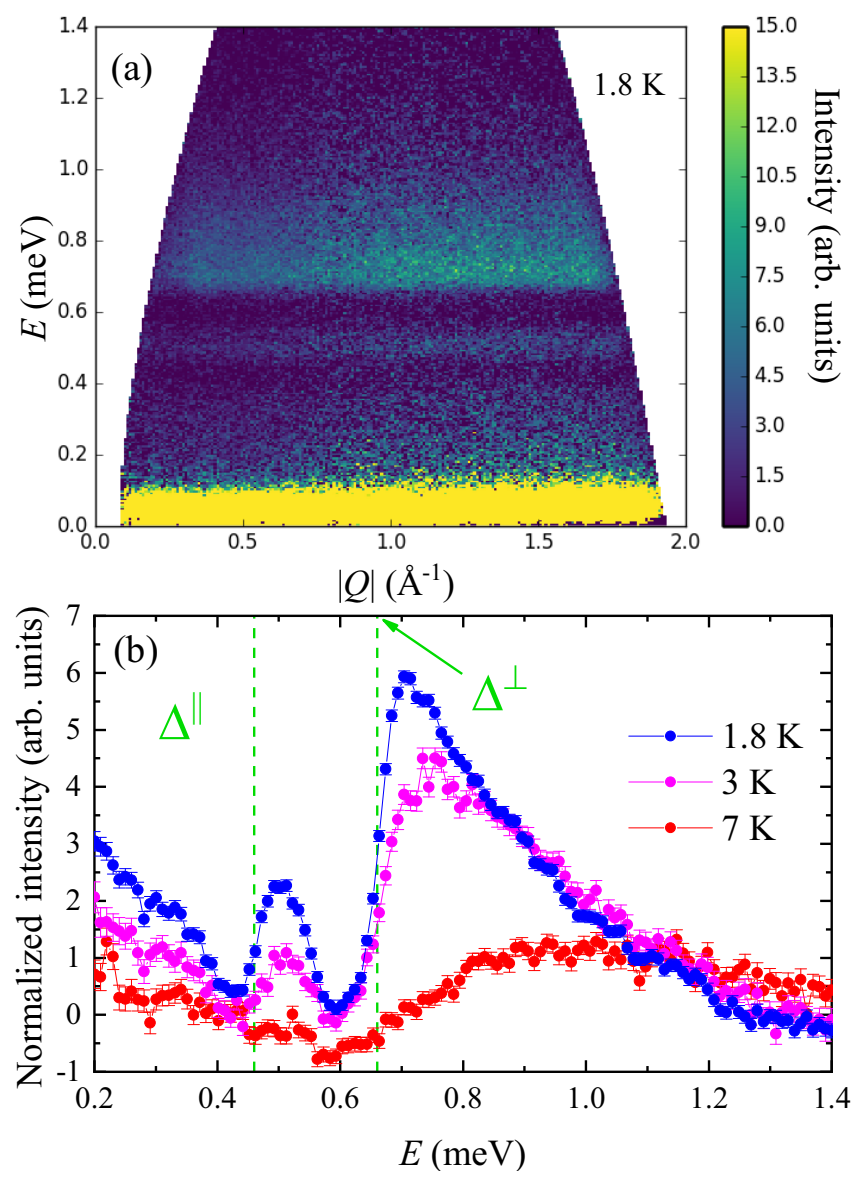

FIG. 4. (a) Time-of-flight INS data, presented as a $(|Q|, E)$ heatmap for $T=1.8 \mathrm{~K}$ and incident neutron energy $E_{\mathrm{i}}=2.2 \mathrm{meV}$, after background subtraction. (b) Representative energy cuts, obtained by integrating over the full measured range of $|Q|$, for the backgroundsubtracted data at $T=1.8,3$, and $7 \mathrm{~K}$.

the first. In terms of intensity, we attribute this difference to the fact that the higher energy mode corresponds to scattering from two degenerate energy bands, while the lower energy feature arises from just one, as shown in the energy level diagram of Fig. 8.

Taking the midpoint of the low-energy onset of each peak yields values for the SIA-split Haldane gaps of $\Delta^{\|}=0.46(1) \mathrm{meV}=5.3(1) \mathrm{K}$ and $\Delta^{\perp}=0.66(1) \mathrm{meV}=$ 7.7(1) $\mathrm{K}$, where labels $\|$ and $\perp$ are assigned using the conclusion that SIA in this system is easy axis in nature, based on the magnetization results discussed above. Using Eq. (2), these gap values correspond to an easy-axis value of the SIA parameter $D=-1.2(1) \mathrm{K}$, in excellent agreement with the estimate based on the anisotropic saturation fields $H_{\mathrm{s}}^{\|, \perp}$. The intrinsic Haldane gap of $\Delta_{0}=0.60(1) \mathrm{meV}=7.0(1) \mathrm{K}$ predicts an intrachain AFM exchange interaction $J=17.0(2)$, where both of these values are in reasonable agreement with the result of the powder magnetometry measurements described above. The ratio of SIA to intrachain exchange is therefore determined to be $D / J=-0.07$ (1) which, together with the absence of LRO for temperatures down to $T=20 \mathrm{mK}$ revealed using muon spin relaxation, places $\mathrm{NiI}_{2}(3,5 \text {-lut })_{4}$ 
firmly in the Haldane phase at low temperatures, as per the phase diagram in Ref. [21].

Muon spin relaxation. Zero-field muon spin-relaxation $\left(\mu^{+} \mathrm{SR}\right)$ measurements were made on a powder sample of $\mathrm{NiI}_{2}(3,5 \text {-lut })_{4}$ to search for LRO in the absence of any applied magnetic field. No oscillations were resolved in the muon spin polarization for temperatures down to $T=20 \mathrm{mK}$, consistent with the absence of magnetic order. Instead, we observe exponential relaxation with very little temperature dependence, implying dynamics in a disordered magnetic-field distribution [59] and no phase transition to an ordered state (see the Appendix). This observation is consistent with the realization of a Haldane state.

To investigate the closure of the Haldane gap, transversefield $\mu^{+}$SR spectra were measured for a powder sample in applied fields $2.0 \leqslant \mu_{0} H_{0} \leqslant 7.0 \mathrm{~T}$ at fixed temperatures $T=0.02 \mathrm{~K}$ and $T=0.75 \mathrm{~K}$. The distribution of local magnetic-field strength $p(B)$ across the sample volume may be obtained by taking the Fourier transform of the measured time-dependent muon spin polarization. Example Fourier spectra for $T=0.02 \mathrm{~K}$, obtained using the maximum entropy method [60], are shown in Fig. 5. The spectra consist of a narrow main peak (common to all) and a low-field shoulder whose character changes with applied field $\mu_{0} H_{0}$. The main peak is due to muons experiencing fields close to $\mu_{0} H_{0}$, in sites in the sample where there are not significant static internal local magnetic fields. This main peak shifts to slightly higher fields as the external field $\mu_{0} H_{0}$ is increased, which could reflect a small (motionally narrowed) paramagnetic contribution to the total local field at these sites due to dilute end chain spins. The broader low-field feature is well coupled to the magnetic behavior, and reflects the contribution from muons experiencing an internal local field in addition to the applied field. A significant local field at the muon site below $\mu_{0} H_{\mathrm{c}}$ is unexpected, but we note that a high-field feature was observed in the $\mu^{+}$SR spectra of the molecular spin-ladder BPCB [61] which was attributed to a muon-induced distortion at the muon site. While both the main peak and the low-field shoulder (and therefore the average spectral weight) shift to higher fields with increasing $\mu_{0} H_{0}$, the shift is larger for the main peak, resulting in increasing separation of the peaks as $\mu_{0} H_{0}$ increases. At $\mu_{0} H_{0} \geqslant 6.0 \mathrm{~T}$ [Fig. 5(e)] the shoulder broadens significantly, which reflects the widening of the local magnetic-field distribution as the field at the muon site increases. The spectra measured at $T=0.75 \mathrm{~K}$ are similar to those measured at $T=0.02 \mathrm{~K}$ [see Fig. 5(e)].

To parametrize the shapes of the field spectra we fit them to the sum of three Gaussian peaks, one to model the main peak, a second to capture the additional weight on the low-field side, and a low amplitude, broad contribution to capture the tails of the main peak:

$$
A(B)=\sum_{i=1}^{3} A_{i} \exp \left[-\frac{\left(B-B_{i}\right)^{2}}{2 \sigma_{i}^{2}}\right] .
$$

The spectral weight (given by the product $A_{i} \sigma_{i}$ ) of the lowfield component is approximately constant, with the peak amplitude decreasing as the feature broadens. The low-field shoulder accounts for $\approx 25 \%$ of the total spectral weight, with the two other components contributing approximately

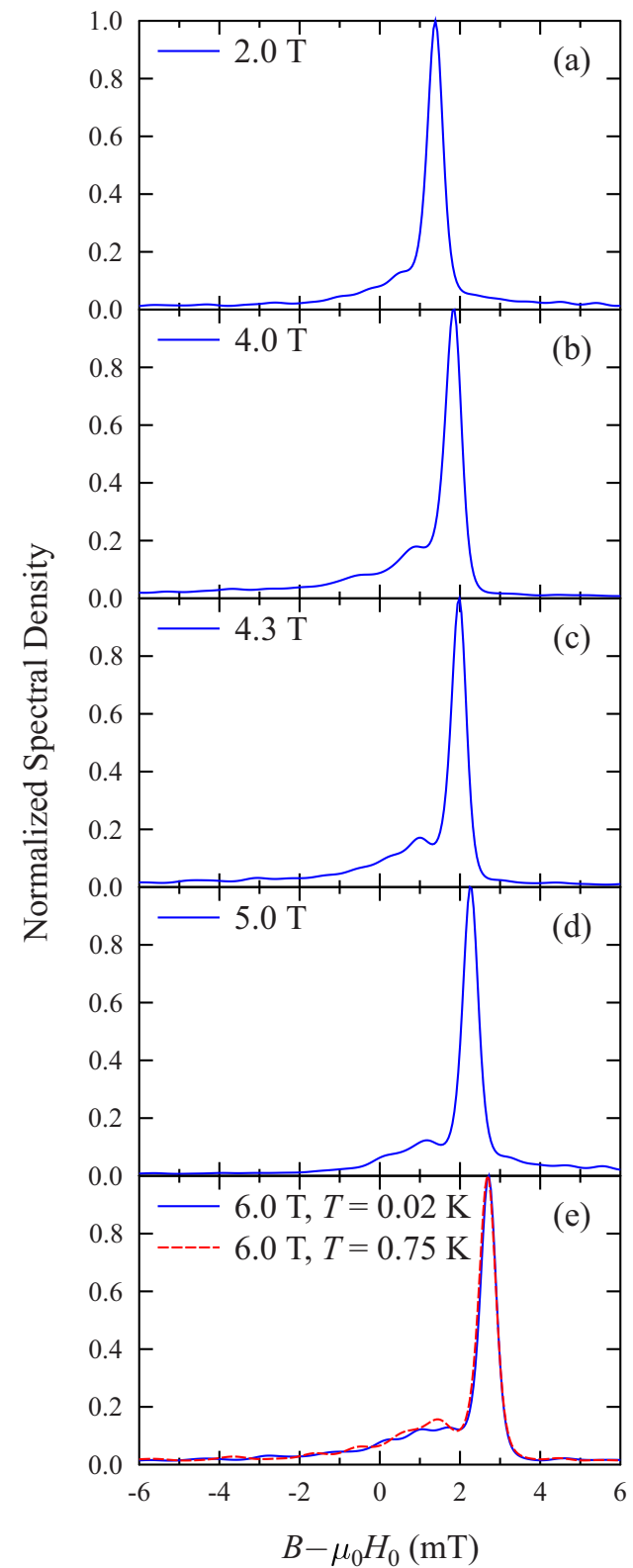

FIG. 5. Fourier transform transverse-field $\mu^{+}$SR spectra measured at $T=0.02 \mathrm{~K}$. In (e) the corresponding spectrum at $T=$ $0.75 \mathrm{~K}$ is shown as the dashed line.

equally to the remaining spectral weight. As seen in Fig. 6, the width of the shoulder is approximately constant up to $\mu_{0} H_{0} \approx$ 4.0 $\mathrm{T}$ beyond which the full width half maximum (FWHM) increases significantly with increasing $\mu_{0} H_{0}$ for both $T=$ $0.02 \mathrm{~K}$ and $T=0.75 \mathrm{~K}$. Similar increases in the width of the field distribution have been seen in $\mu^{+} \mathrm{SR}$ experiments on the molecular magnet $[\mathrm{Cu}$ (pyrazine) (glycinate) $]\left(\mathrm{ClO}_{4}\right)$ [9] and in the candidate Bose-Einstein condensation material $\mathrm{Pb}_{2} \mathrm{~V}_{3} \mathrm{O}_{9}$ [62], where rapid rises in the relaxation rate were observed at field-induced transitions. This suggests that the system undergoes a crossover in behavior or phase transition for fields $\mu_{0} H_{0} \gtrsim 4.0 \mathrm{~T}$.

The solid line in Fig. 6(a) is a guide to the eye, capturing the field dependence of the low-field peak FWHM for 


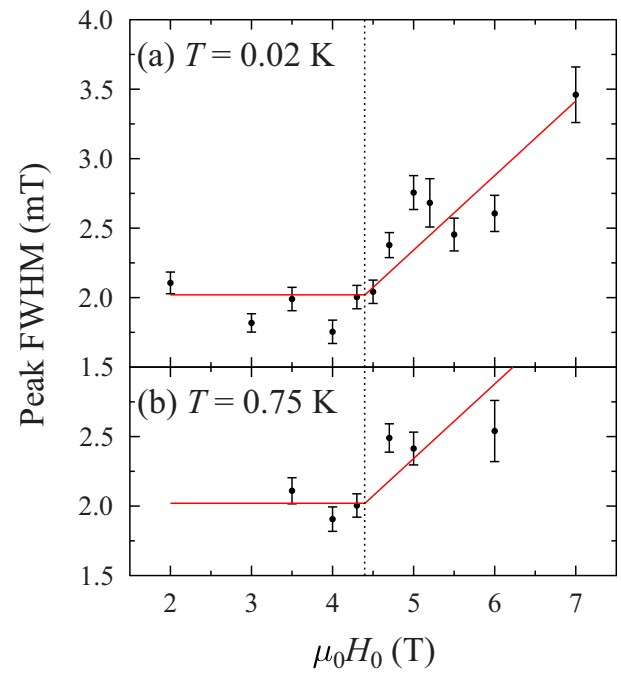

FIG. 6. Full width half maximum (FWHM) for the low-field peak at (a) $T=0.02 \mathrm{~K}$ and (b) $T=0.75 \mathrm{~K}$. The solid lines are guides to the eye, and are the same for both temperatures.

$T=0.02 \mathrm{~K}$. We estimate $\mu_{0} \bar{H}_{\mathrm{c}} \approx 4.4(2) \mathrm{T}$ for the critical field, in agreement with the powder magnetization results. The same solid line is overlaid with the data for $T=0.75 \mathrm{~K}$ in Fig. 6(b), showing that the behavior of the peak FHWM is similar at both temperatures.

Electron spin resonance. In order to further elucidate the nature of the axial SIA induced by the $\mathrm{Ni}$ (II) tetragonal local environment, ESR measurements were performed on a powder sample of $\mathrm{NiI}_{2}(3,5 \text {-lut })_{4}$. Transmission spectra collected in the first-derivative mode at high temperature are presented in Fig. 7(a), where $T=30 \mathrm{~K}$ is sufficiently high, relative to the energy scales of the intrachain exchange coupling $J$ and resultant Haldane gap $\Delta_{0}$, that the system is within the thermally disordered phase. The spectra display a single exchange-narrowed transition and are therefore not indicative of the value of $D$. This has been seen elsewhere, for example in the related compound $\mathrm{PbNi}_{2} \mathrm{~V}_{2} \mathrm{O}_{8}$ [45]. A linear fit to the transition positions in the field-frequency plane is shown as a dashed line in Fig. 7(a), which yields the powder-average $g$ factor $g=2.19(1)$, in close agreement with the value obtained using magnetic susceptibility.

At low temperatures $(T=3 \mathrm{~K})$, the Haldane singlet phase is the ground state for applied magnetic fields below the anisotropic critical values $\mu_{0} H_{\mathrm{c}}^{\|}=5.3 \mathrm{~T}$ and $\mu_{0} H_{\mathrm{c}}^{\perp}=4.3 \mathrm{~T}$, whereupon a triplet state is driven lower in energy. ESR transitions between the singlet Haldane phase and triplet states are forbidden by momentum conservation, and are not observed in our data. The central feature which dominates at low frequencies [indicated by green arrows and circles in Figs. 7(b) and 7(c), respectively] is ascribed to fractional $S=$ $1 / 2$ end chain degrees of freedom and is therefore considered separately to all other observed resonances. This resonance is still observed for fields above the critical values, as seen in the Haldane compound NENB, for example [63], albeit with a suppressed amplitude as the Haldane state depopulates for $H>H_{\mathrm{c}}$. The resonance positions are fit to an isotropic model for $S=1 / 2$ moments [green line in Fig. 7(c), which yields the

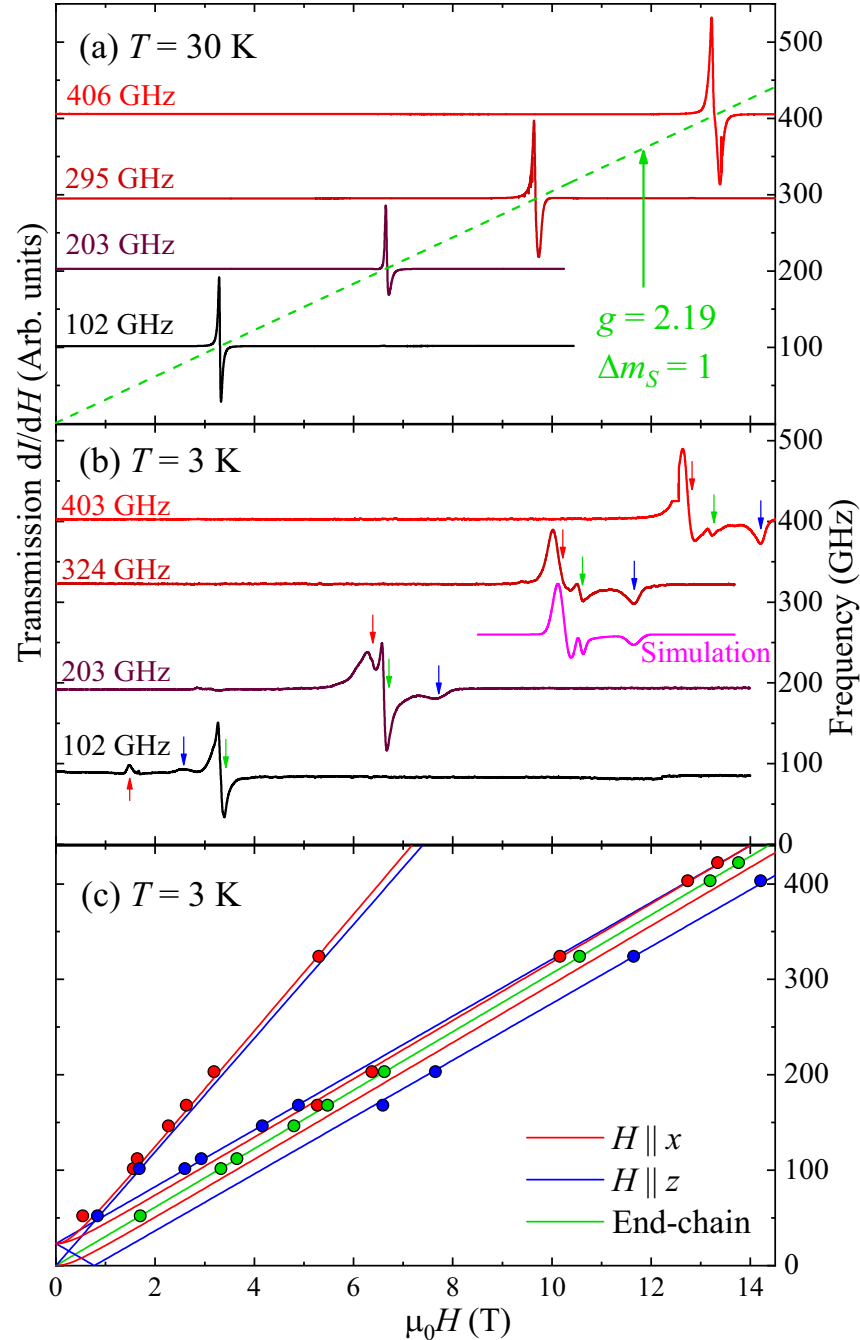

FIG. 7. The frequency dependence of ESR transmission spectra for a powder sample of $\mathrm{NiI}_{2}(3,5-\mathrm{lut})_{4}$ at (a) $T=30 \mathrm{~K}$ and (b) $T=3 \mathrm{~K}$. (c) The positions of transitions observed at $T=3 \mathrm{~K}$. The resonance branches whose frequency change most rapidly with applied field correspond to the half-field transitions, while the other intratriplet transitions appear as low- and high-field satellites to the end chain resonances. Examples of these $S=1$ transitions are labeled $\alpha, \beta$, and $\gamma$ in Fig. 8. The dashed line in (a) and solid lines in (c) are fits described in the text and the simulated spectrum in (b) is obtained with $v=324 \mathrm{GHz}$.

$g$ factor $g=2.191(4)$, consistent with the high-temperature result for the bulk $S=1$ moments.

The remaining resonances observed at $T=3 \mathrm{~K}$ are indicated by red and blue arrows and circles in Figs. 7(b) and 7(c), and comprise satellite features on the low- and high-field side of the central resonance. Examples of these transitions for $H \| x$ are labeled $\beta$ and $\gamma$ respectively on the energy-level diagram shown in Fig. 8. Also observed are the "half-field" resonances corresponding to transitions with $\Delta m_{S}= \pm 2$ [64] (labeled $\alpha$ in Fig. 8). At low frequencies and fields $(H \lesssim$ $\left.H_{\mathrm{c}}\right)$ there are fewer transitions between excited triplet states, resulting in small amplitudes for the satellite peaks relative to the central end chain resonance. However, for fields above the 


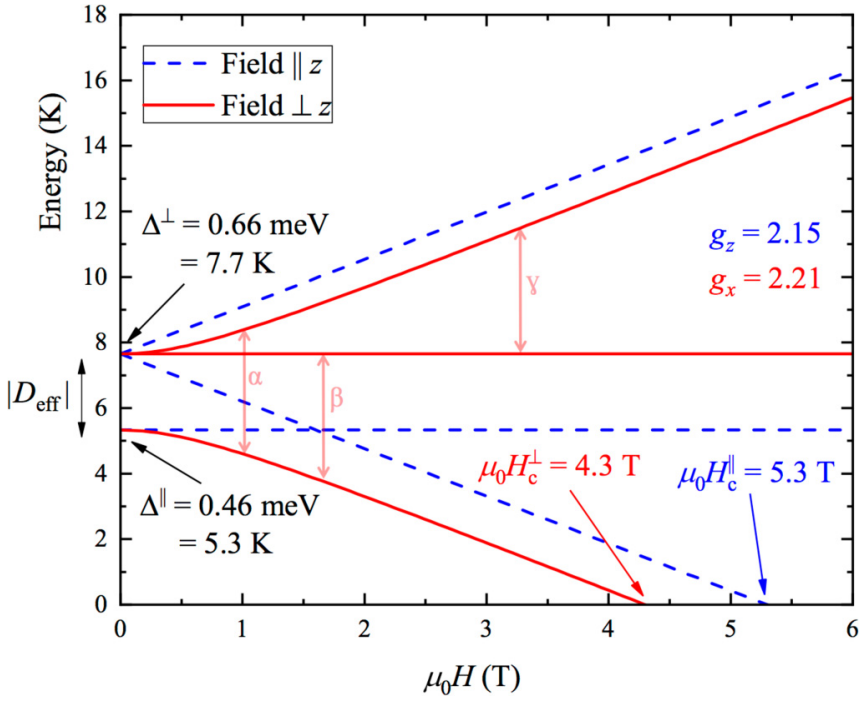

FIG. 8. The anisotropic field dependence of energy levels within the Haldane phase, according to the fermion model. The anisotropic critical fields and zero-field energy gap values have been set to the values determined by magnetization (Fig. 3) and INS (Fig. 4), respectively. The $g$ factors have been fixed to the values for the fermion model from Table I, in order to satisfy the relations in Eq. (4). The effective SIA term $D_{\text {eff }}=+2.4$ (2) K defined by Eq. (3) is labeled. Examples of the transitions observed in the ESR data are labeled as $\alpha, \beta$, and $\gamma$.

critical value a low-energy triplet state becomes the ground state, and these resonances rapidly gain intensity.

Based on their line shapes [64] (peak versus peak derivative), the noncentral transitions are ascribed to fields parallel to the $z$ direction (color-coded blue) and the $x$ direction (red). These positions may be fitted to the fermion model, which is equivalent to the description of isolated moments possessing axial SIA [41]. This procedure captures the positions of the resonances, as shown by the blue and red lines in Fig. 7(c), and yields the anisotropic $g$ factors $g_{\|}=g_{z}=2.13(1)$ and $g_{\perp}=g_{x}=2.19(1)$, and an easy-plane effective SIA parameter $D_{\text {eff }}=+1.11(6) \mathrm{K}$, and hence an easy-axis $D$ in the Hamiltonian [see Eqs. (1) and (3)]. A simulated spectrum created by summing the two contributions outlined above is compared to the data measured for $v=324 \mathrm{GHz}$ in Fig. 7(b), where it may be seen that the line shapes and positions of the main resonances (and the relative intensities of the two satellite peaks) are well described by the simulation.

\section{DISCUSSION}

The INS and single-crystal magnetization studies provide direct measurements of the SIA-split Haldane gaps and anisotropic critical fields, respectively, for $\mathrm{NiI}_{2}(3,5-\mathrm{lut})_{4}$. These quantities can be related via the model-dependent expressions given for the fermion and boson theories in Eqs. (4) and (5), respectively. In order to test the validity of these theories for this system, the anisotropic $g$ factors that each model predicts, given the experimentally observed values of $\Delta^{\|, \perp}$ and $H_{\mathrm{c}}^{\|, \perp}$, are shown in Table I. The two models yield an identical value of $g_{\|}=2.15(5)$ which is
TABLE I. The anisotropic and powder $g$ factors predicted by the fermion [Eqs. (4)] and boson [Eqs. (5)] models, using the values of $\Delta^{i}$ and $H_{\mathrm{c}}^{i}$ directly observed using INS and magnetization, respectively.

\begin{tabular}{llcc}
\hline \hline Theory & $g_{\|}=g_{z}$ & $g_{\perp}=g_{x}$ & $g_{\text {powder }}$ \\
\hline Fermion & $2.15(5)$ & $2.21(6)$ & $2.19(4)$ \\
Boson & $2.15(5)$ & $1.85(6)$ & $1.95(4)$ \\
\hline \hline
\end{tabular}

physically reasonable for $\mathrm{Ni}$ (II) ions, and in close agreement with the value determined using the low-temperature ESR measurements. The fermion value of $g_{\perp}=2.21(6)$ and the resultant powder average $g_{\text {powder }}=\left(g_{\|}+2 g_{\perp}\right) / 3=2.19(4)$ are also in excellent agreement with the high- and lowtemperature ESR and powder susceptibility results. However, the boson value of $g_{\perp}=1.85(6)$ is smaller than is plausible for this ion, and the powder average value $g_{\text {powder }}=1.95(4)$ is not in good agreement with the aforementioned techniques.

The fermion model is therefore in excellent agreement with the experimental results for the Haldane phase of $\mathrm{NiI}_{2}(3,5-\mathrm{lut})_{4}$, demonstrating that its applicability is not limited to easy-plane Haldane compounds, as has been recently suggested [46,47]. The resultant energy levels are shown in Fig. 8, where the values of $g_{\|, \perp}$ from Table I reproduce the experimentally observed energy gaps $\Delta^{\|, \perp}$ and critical fields $H_{\mathrm{c}}^{\|, \perp}$. The boson model has previously been successful in describing compounds with critical interchain interactions $[36,46,47]$, and its failure to consistently describe the experimental findings for $\mathrm{NiI}_{2}(3,5-\mathrm{lut})_{4}$ may constitute further evidence for small $\left|J_{\perp}\right|$ in this compound.

INS provides a direct, zero-field measurement of the SIAsplit triplet energy levels, and therefore unequivocally yields $D=-1.2(1) \mathrm{K}$ [and $\left.\left.D_{\text {eff }}=+2.4(2) \mathrm{K}\right)\right]$, in excellent agreement with the estimate based on the anisotropic saturation behavior probed using pulsed-field magnetometry. Interestingly, the fermion model appears to describe powder ESR data for transitions between triplet states under the application of magnetic field, but with $D_{\text {eff }}=+1.11(6) \mathrm{K}$, which is close to half of the INS value. This discrepancy could be due to the fact that the energy levels of the Haldane chain are not well understood for fields above the critical field $H_{\mathrm{c}}$ [65]. The fermion and boson models have been shown to fail in the description of energy levels above $H_{\mathrm{c}}$, albeit in systems which undergo three-dimensional magnetic ordering above the critical field. In the related Haldane compounds $\mathrm{SrNi}_{2} \mathrm{~V}_{2} \mathrm{O}_{8}$ and NDMAP, INS $[44,47,66]$ and ESR $[65,67]$ have demonstrated that the triplet energy levels change gradient as magnetic field is swept through $H_{\mathrm{c}}$, in contrast to the prediction of the fermion model.

The intrachain exchange coupling $J=17.5(2) \mathrm{K}$ is deduced from the saturation behavior observed in the pulsedfield magnetization measurements, while the INS measurement yields $\Delta_{0}=7.0(1) \mathrm{K}$. The resulting ratio $\Delta_{0} / J=$ 0.40 (1) is very close to the value of 0.41 expected for an ideal isolated chain, implying that the interchain exchange should not play a significant role in our material, at least at the temperatures explored here. In contrast, the SIA-split Haldane gaps in $\mathrm{PbNi}_{2} \mathrm{~V}_{2} \mathrm{O}_{8}$ and $\mathrm{SrNi}_{2} \mathrm{~V}_{2} \mathrm{O}_{8}$ are driven lower in energy by substantial interchain dispersion due to $J_{\perp}$ [19]. 
TABLE II. A comparison of parameter values for selected Haldane compounds.

\begin{tabular}{lccccccccc}
\hline \hline Compound & $J(\mathrm{~K})$ & $D(\mathrm{~K})$ & $D / J$ & $\Delta^{\|}(\mathrm{K})$ & $\Delta^{\perp}(\mathrm{K})$ & $\Delta_{0} / J$ & $H_{\mathrm{c}}^{\|}(\mathrm{T})$ & $H_{\mathrm{c}}^{\perp}(\mathrm{T})$ & $\mathrm{References}$ \\
\hline $\mathrm{NiI}_{2}(3,5-\mathrm{lut})_{4}$ & $17.5(2)$ & $-1.2(1)$ & $-0.07(1)$ & $5.3(1)$ & $7.7(1)$ & $0.40(1)$ & $5.3(1)$ & $4.3(1)$ & this study \\
$\mathrm{PbNi}_{2} \mathrm{~V}_{2} \mathrm{O}_{8}$ & 104 & -5.2 & -0.05 & 21 & 28 & 0.25 & 19.0 & 14 & {$[18,19,45]$} \\
$\mathrm{SrNi}_{2} \mathrm{~V}_{2} \mathrm{O}_{8}$ & 101 & -3.7 & -0.04 & 18.2 & 29.9 & 0.26 & 20.8 & 12.0 & {$[46,53]$} \\
$\mathrm{Y}_{2} \mathrm{BaNiO}_{5}$ & 280 & -9.4 & -0.03 & 87 & 100,110 & 0.35 & $\sim 70$ & {$[68,70]$} \\
$\mathrm{AgVP}_{2} \mathrm{~S}_{6}$ & 780 & 4.5 & 0.006 & & 300 & 0.38 & {$[200$} & {$[57]$} \\
\hline \hline
\end{tabular}

Hence $\mathrm{NiI}_{2}(3,5 \text {-lut })_{4}$ is remarkable as a highly isolated Haldane chain with a single crystallographic $\mathrm{Ni}$ (II) site, experimentally accessible energy scales $\left(J=17.5(2) \mathrm{K}, \Delta^{\|, \perp}=\right.$ $\left.5.3,7.7 \mathrm{~K}, H_{\mathrm{c}}^{\|, \perp}=5.3,4.3 \mathrm{~T}\right)$ and an anisotropy ratio $D / J=$ $-0.07(1)$. Systems with comparable degrees of anisotropy include $\mathrm{Y}_{2} \mathrm{BaNiO}_{5}$ [68], $\mathrm{PbNi}_{2} \mathrm{~V}_{2} \mathrm{O}_{8}$, and $\mathrm{SrNi}_{2} \mathrm{~V}_{2} \mathrm{O}_{8}$ [19], while $\mathrm{AgVP}_{2} \mathrm{~S}_{6}$ is virtually isotropic [69]. The parameters of all five compounds are compared in Table II. The large energy scales exhibited by both $\mathrm{Y}_{2} \mathrm{BaNiO}_{5}$ and $\mathrm{AgVP}_{2} \mathrm{~S}_{6}$ preclude field-tuning through the quantum critical region associated with the closure of their Haldane gaps using dc magnetic fields. While $H_{\mathrm{c}}^{\perp}$ for $\mathrm{PbNi}_{2} \mathrm{~V}_{2} \mathrm{O}_{8}$ and $\mathrm{SrNi}_{2} \mathrm{~V}_{2} \mathrm{O}_{8}$ are within the range of superconducting magnets, both systems have near-critical values of $\left|J_{\perp}\right| / J$, lying on the very edge of the Haldane phase as evidenced by the departure of $\Delta_{0} / J$ from the ideal value of 0.41 and the observation of long-range magnetic order at liquid-helium temperatures in applied fields. $\mathrm{NiI}_{2}(3,5-\mathrm{lut})_{4}$ is therefore a uniquely ideal Haldane system that provides easy access to the entire Haldane phase for all field directions. In particular, the entire quantum critical region can be probed with techniques that may have hitherto not been viable for other compounds. This includes instruments at central beamtime facilities, as our preliminary $\mu^{+} \mathrm{SR}$ investigations demonstrate.

Furthermore, the molecular building blocks that make up $\mathrm{NiI}_{2}$ (3, 5-lut) 4 permit a higher degree of controlled structural modification than is possible for the inorganic systems. Of particular interest is the controlled introduction of quenched bond disorder. Randomly distributed nonmagnetic defects on the transition-metal sites and substitutions which locally modify superexchange pathways have proven to be a route to exciting physics [71], yet still represent an unsolved problem. Controlled chemical substitution in related molecule-based materials has been demonstrated to give rise to exotic phases, such as the elusive Bose-glass phase in the large- $D$ system DTN [72] and the spin-ladder IPA- $\mathrm{CuCl}_{3}$ [73]. Bond disorder in the Haldane phase has been considered through numerical simulations, which predict localized impurity bound states $[74,75]$. However, to date, the paucity of physically realized compounds where controlled introduction of bond disorder can be achieved has led to a lack of experimental verification of these theoretical predictions.

\section{CONCLUSIONS}

In summary, we have shown that $\mathrm{NiI}_{2}(3,5 \text {-lut })_{4}$ is a realization of a $S=1$ Heisenberg AFM 1D chain, with intrachain exchange coupling $J=17.5$ (2) $\mathrm{K}$ manifested by unique Ni-I ... I-Ni magnetic couplings. Muon spin-relaxation measurements demonstrate the absence of zero-field magnetic
LRO down to $T=20 \mathrm{mK}$, indicating a high degree of one dimensionality (i.e., a small ratio $\left|J_{\perp}\right| / J$ ). The results of INS measurements prove this compound resides within the Haldane region of the theoretical phase diagram from Ref. [21], and quantify the Haldane energy gaps for the SIA-split triplet excitations $\Delta^{\|}=0.46(1) \mathrm{meV}$ and $\Delta^{\perp}=$ $0.66(1) \mathrm{meV}$. These energy gaps correspond to an easy-axis SIA parameter $D=-1.2(1) \mathrm{K}$, so that $D / J=-0.07(1)$, making this one of the most isotropic Haldane systems reported to date. Single-crystal magnetization measurements reveal the anisotropic critical fields $\mu_{0} H_{\mathrm{c}}^{\|}=5.3(1) \mathrm{T}$ and $\mu_{0} H_{\mathrm{c}}^{\perp}=4.3$ (1) $\mathrm{T}$, which are readily accessible in commercially available superconducting magnets, and powder pulsedfield measurements demonstrate the system is saturated at $\mu_{0} H_{\mathrm{s}}^{\|}=46.0(4) \mathrm{T}$ and $\mu_{0} H_{\mathrm{s}}^{\perp}=50.7(8) \mathrm{T}$. These energy gaps and critical fields are explicable using the fermion model, in contrast to other reported easy-axis Haldane systems where $J_{\perp}$ is critically large and the boson model is more consistent with experiment [45-47].

$\mathrm{NiI}_{2}(3,5 \text {-lut })_{4}$ is also ideally suited to the introduction of quenched disorder via substitution of the $\mathrm{Ni}$ (II) ion and the halide ions which mediate the superexchange interaction. This model system therefore provides a unique opportunity to explore this relatively uncharted territory and gain fresh insight into the physics of the Haldane phase using chemistry to control and tune the exchange interactions and SIA.

CCDC 1960981 contains the supplementary crystallographic data for this paper. These data can be obtained free of charge from The Cambridge Crystallographic Data Centre [76]. Data presented in this paper resulting from the UK effort will be made available [77]. Furthermore, all raw neutron data and associated metadata obtained as a result of access to ISIS, reside in the public domain, with ISIS acting as the custodian. The data can be accessed from [78].

\section{ACKNOWLEDGMENTS}

We thank J. Liu and M. Coak for useful discussions. This project has received funding from the European Research Council (ERC) under the European Union's Horizon 2020 research and innovation program (Grant Agreement No. 681260). W.J.A.B. thanks the EPSRC for additional support. A portion of this work was performed at the National High Magnetic Field Laboratory, which is supported by National Science Foundation (NSF) Cooperative Agreements No. DMR-1157490 and No. DMR-1644779, the State of Florida and the U.S. Department of Energy (DOE) through the Basic Energy Science Field Work Proposal "Science in 100 T". Work at EWU was supported by NSF Grant 
No. DMR-1703003. NSF's ChemMatCARS Sector 15 is principally supported by the Divisions of Chemistry (CHE) and Materials Research (DMR), NSF, under Grant No. NSF/CHE-1834750. Use of the Advanced Photon Source, an Office of Science User Facility operated for the U.S. DOE Office of Science by Argonne National Laboratory, was supported by the U.S. DOE under Contract No. DEAC02-06CH11357. J.A.S. acknowledges support from the Independent Research/Development (IRD) program while serving at the National Science Foundation. F.X. acknowledges funding from the European Union's Horizon 2020 research and innovation program under the Marie SkodowskaCurie Grant Agreement No 701647. A portion of this work was performed at the Swiss Muon Source, Paul Scherrer Institute, Switzerland and we acknowledge support from EPSRC under Grants No. EP/N023803/1, No. EP/N024028/1, and No. EP/N032128/1. B.M.H. thanks STFC for support via a studentship.

\section{APPENDIX: EXPERIMENTAL DETAILS}

\section{Synthesis}

All chemical reagents were purchased from commercial sources and used as received. A typical synthesis involves slow mixing of aqueous solutions of $\mathrm{Ni}\left(\mathrm{NO}_{3}\right)_{2}$. $6 \mathrm{H}_{2} \mathrm{O}(0.4002 \mathrm{~g}, 1.38 \mathrm{mmol})$ with excess $\mathrm{NH}_{4} \mathrm{I}(0.9971 \mathrm{~g}$, $6.88 \mathrm{mmol})$. In 5 -mL of acetonitrile, $2-\mathrm{mL}$ of 3,5-lutidine was dissolved and slowly added by pipette to the aqueous Ni solution. The resulting solution is mostly blue with a slightly green hue. Slow solvent evaporation over the period of a few days resulted in yellow-green microcrystals. Larger single crystals can be grown using a vapor diffusion method wherein 3,5-lutidine is allowed to slowly diffuse into an aqueous solution containing $\mathrm{Ni}\left(\mathrm{NO}_{3}\right)_{2} \cdot 6 \mathrm{H}_{2} \mathrm{O}$ and $\mathrm{NH}_{4} \mathrm{I}$. In either case, crystals of the product are obtained in better than $90 \%$ yield. The crystals were found to be largely air stable although they were generally stored in a refrigerator.

\section{Structural determination}

Experiments were conducted on the ChemMatCARS 15ID-B beamline of the Advanced Photon Source at Argonne National Laboratory. A microcrystal of $\mathrm{NiI}_{2}(3,5-\mathrm{lut})_{4}$ measuring $10 \times 10 \times 2 \mu \mathrm{m}^{3}$ was selected from a bulk sample using a cryoloop and mounted on a Huber three-circle $\mathrm{x}$-ray diffractometer equipped with an APEX II charge-coupled device area detector. The sample was cooled to 100(2) K using a $\mathrm{LN}_{2}$ cryojet. Synchrotron radiation with a beam energy of $32.2 \mathrm{keV}(\lambda=0.41328 \AA)$ was selected and the beam size at the sample position was $0.1 \times 0.1 \mathrm{~mm}^{2}$. The distance between sample and detector was set at $60 \mathrm{~mm}$. A total of 51036 reflections were collected of which 1272 were unique $[I>2 \sigma(I)]$.

Data collection and integration were performed using the APEX II software suite. Data reduction employed SAINT [79]. Resulting intensities were corrected for absorption by Gaussian integration (SADABS) [80]. The structural solution (XT) [81] and refinement (XL) [82] were carried out with SHELX software using the XPREP utility for the space-group determination. Considering systematic absences, the crystal structure
TABLE III. Parameters related to the structural refinement of the x-ray data. The weighted $R$ factor $w R$ and goodness of fit (GOF) are based on $F^{2}$ for the threshold $F^{2}>2 \sigma\left(F^{2}\right)$.

\begin{tabular}{lc}
\hline \hline Parameter (units) & Fit results (error) \\
\hline Instrument & Huber 3-circle fixed-Kappa \\
Method & single crystal \\
$T(\mathrm{~K})$ & 100 \\
$\lambda(\AA)$ & 0.41328 \\
Emp. formula & $\mathrm{C}_{28} \mathrm{H}_{36} \mathrm{I}_{2} \mathrm{~N}_{4} \mathrm{Ni}$ \\
Formula mass $\left(\mathrm{g} \mathrm{mol}^{-1}\right)$ & 741.11 \\
Crystal class & tetragonal \\
Space group & $P 4 / n n c$ \\
$a(\AA)$ & $12.0019(3)$ \\
$b(\AA)$ & $12.0019(3)$ \\
$c(\AA)$ & $9.9783(2)$ \\
$\alpha\left(^{\circ}\right)$ & 90 \\
$\beta\left({ }^{\circ}\right)$ & 90 \\
$\gamma\left({ }^{\circ}\right)$ & 90 \\
$V\left(\AA^{3}\right)$ & $1437.33(8)$ \\
$Z$ & 2 \\
$\rho_{\text {exp }}\left(\mathrm{g} \mathrm{cm}{ }^{-3}\right)$ & 1.712 \\
$\mu\left(\mathrm{mm}^{-1}\right)$ & 0.646 \\
No. meas. refs. & 51036 \\
No. uniq. refs. & 1272 \\
No. params. & 44 \\
$\mathrm{GOF}$ & 1.184 \\
$R_{1}$ & 0.0142 \\
$w R_{2}$ & 0.0420 \\
$\Delta \rho \pm\left(e \AA^{-3}\right)$ & $0.611,0.476$ \\
\hline \hline
\end{tabular}

was solved in the tetragonal space group $P 4 / n n c$ (no. 126) [83]. Lutidine $\mathrm{H}$ atoms were placed in idealized positions and allowed to ride on the carbon atom to which they are attached. All nonhydrogen atoms were refined with anisotropic thermal displacement parameters.

Table III shows the results of the structural refinement.

\section{Magnetometry}

SQUID. Quasistatic magnetometry measurements were performed using a Quantum Design SQUID magnetometer in fields up to $7 \mathrm{~T}$, where an iQuantum ${ }^{3} \mathrm{He}$ insert enabled temperatures $T \gtrsim 0.5 \mathrm{~K}$ to be reached. The powder and crystal samples were loaded into a gelatin capsule, which was then attached to a nonmagnetic sample holder at the end of a rigid rod. The temperature-dependent molar magnetic susceptibility is obtained in the linear limit via $\chi_{\mathrm{m}}=M /(n H)$ where the powder contains $n$ moles of the compound.

It is found that components of the iQuantum ${ }^{3} \mathrm{He}$ insert produce a background signal, which can be significant for small sample signals. In our case this is the situation at low temperatures and low fields, where the majority $S=1$ spins of the Haldane chain are gapped. In this region the intrinsic susceptibility and magnetization are expected to be near zero, with the only contribution arising from the small proportion of $S=1 / 2$ end chain excitations that are observed in the ESR measurements. Problems arise when attempting to accurately subtract the background response from the data to obtain the sample signal in this region. One difficulty is reproducibly 

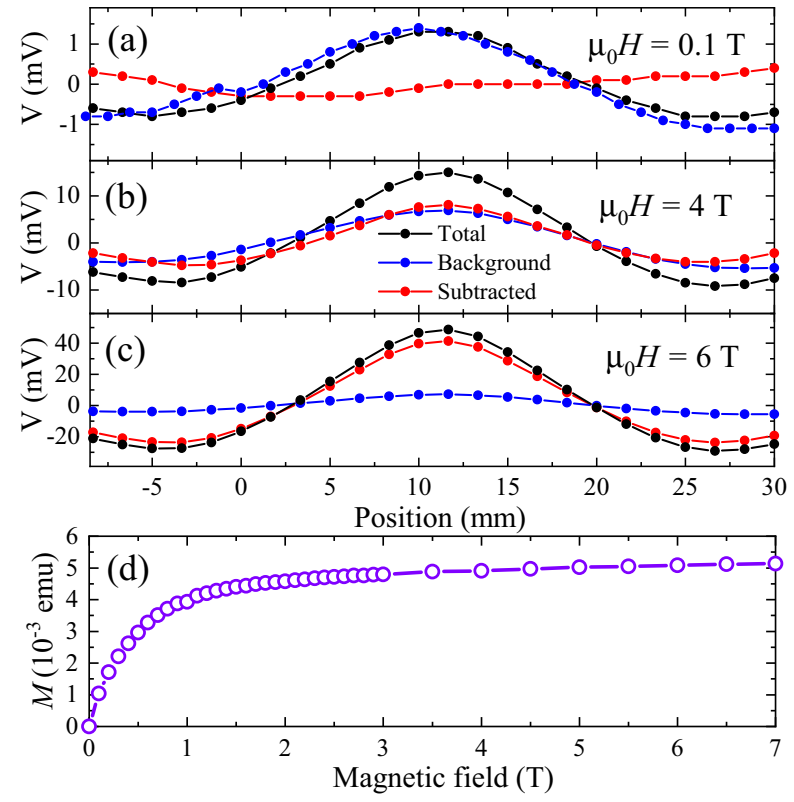

FIG. 9. Raw SQUID dipole signal vs position plots for measurements using the iQuantum ${ }^{3} \mathrm{He}$ insert at $0.5 \mathrm{~K}$ showing the total (sample + background) signal, example background signal and the background-subtracted result at (a) $0.1 \mathrm{~T}$ in the Haldane-gapped phase (b) $4 \mathrm{~T}$ close to the closure of the Haldane gap and (c) $6 \mathrm{~T}$ once the gap is closed. Background subtraction is performed using the SquidLab program [87]. (d) Measured background only $M(H)$ response at $0.5 \mathrm{~K}$.

positioning both the iQuantum insert and the sample within the insert relative to the SQUID measurement coils. It is known from experiments using other high-background SQUID inserts (e.g., rotators and pressure cells) or tiny samples [84-86] that small misalignments in such measurements can have a significant effect on the resulting backgroundsubtracted data for samples with small signals. For these reasons we have found that we cannot reliably remove the background from the iQuantum ${ }^{3} \mathrm{He}$ insert for the measurements of $\chi(T)$ below $1.8 \mathrm{~K}$, and $M(H)$ at fields much less than $4 \mathrm{~T}$ for our sample.

The relative sizes of the background and sample signals are illustrated in Fig. 9, which shows the raw response measured by the SQUID at $0.5 \mathrm{~K}$ and (a) $0.1 \mathrm{~T}$, (b) $4 \mathrm{~T}$, and (c) $6 \mathrm{~T}$ as the insert plus sample is moved between the measurement coils. The response of the background, which is large compared to the sample signal at low fields, is seen to be that of a dipole, similar to the response of a magnetic material. For the lowfield measurement shown in Fig. 9(a), the remaining signal after background subtraction is largely flat despite the presence of paramagnetic spins in the material. Furthermore, on removal of the sample and reinsertion of the iQuantum insert, the location of the center of the background signal can move a small amount relative to the measurement coils, despite best efforts to reproduce the position, leading to irreproducibility in the results. However, at higher fields, particularly above the field where the Haldane gap closes, the sample signal becomes significantly larger than the background as shown in Figs. 9(b) and 9(c). A measurement of the background $M(H)$ alone (i.e., without sample) is shown in Fig. 9(d). It is seen that the response resembles that of a collection of paramagnetic spins, which is similar to the response expected from the sample due to end chains (and possible paramagnetic impurities). Indeed the total (sample + background) $T=0.5 \mathrm{~K}$ response between 0 and $2 \mathrm{~T}$ can be well modelled by an $S=1 / 2$ Brillouin function, as shown in Fig. 2(b). The background $M(H)$ response becomes relatively flat for fields in excess of $1.5 \mathrm{~T}$ as the background magnetization becomes saturated, and above this field the background provides a constant offset to the data when measured in combination with the sample. At fields approaching the critical field for closure of the Haldane gap the sample dominates the measured signal, as suggested by the raw scans discussed above, and the positions of the critical fields in both the powder and single-crystal experiments are not affected by the background.

Pulsed fields. Isothermal pulsed-field magnetization measurements were performed at the National High Magnetic Field Laboratory in Los Alamos, USA. Fields of up to $65 \mathrm{~T}$ with typical rise times $\approx 10 \mathrm{~ms}$ were used, and a ${ }^{3} \mathrm{He}$ cryostat provides temperature control. A powdered sample was mounted in a $1.3-\mathrm{mm}$-diameter PCTFE ampoule which was attached to a probe containing a 1500-turn, $1.5-\mathrm{mm}$ bore, 1.5 $\mathrm{mm}$-long compensated-coil susceptometer, constructed from
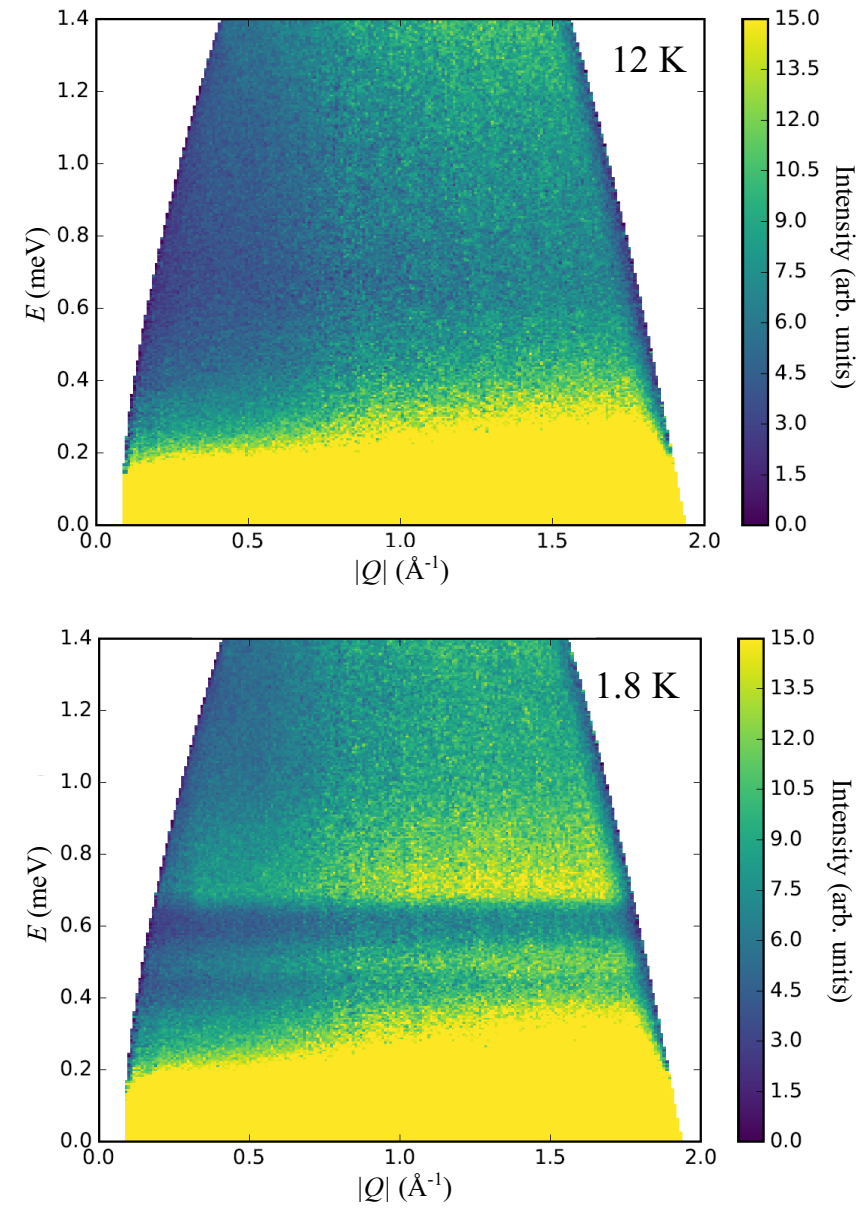

FIG. 10. Time-of-flight INS data, presented as a $(|Q|, E)$ heat map for $T=12 \mathrm{~K}$ (top) and $T=1.8 \mathrm{~K}$ (bottom) and incident neutron energy $E_{\mathrm{i}}=2.19 \mathrm{meV}$. Figure 4 shows the $12-\mathrm{K}$ data subtracted from the $1.8 \mathrm{~K}$. 
(a)
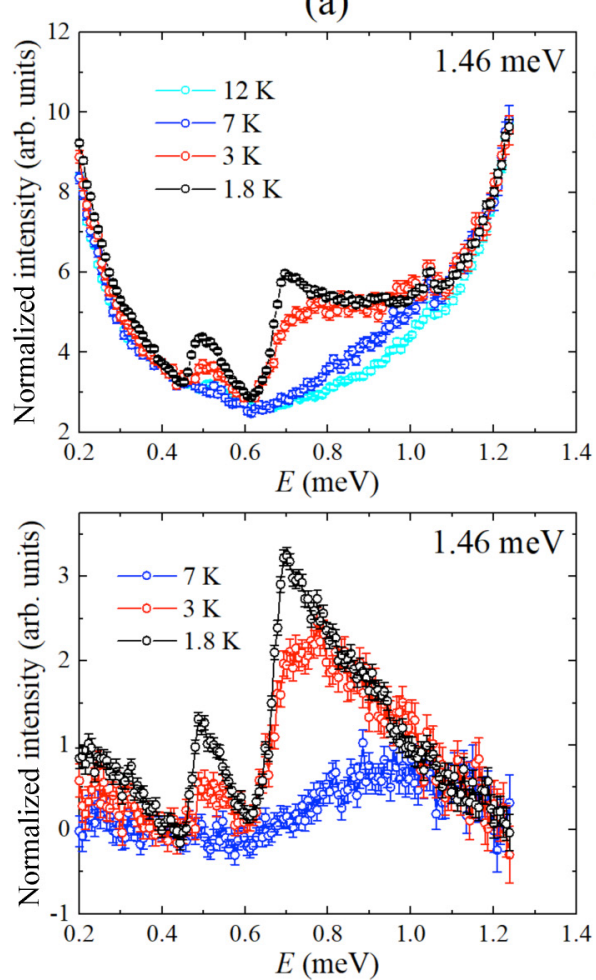

(b)
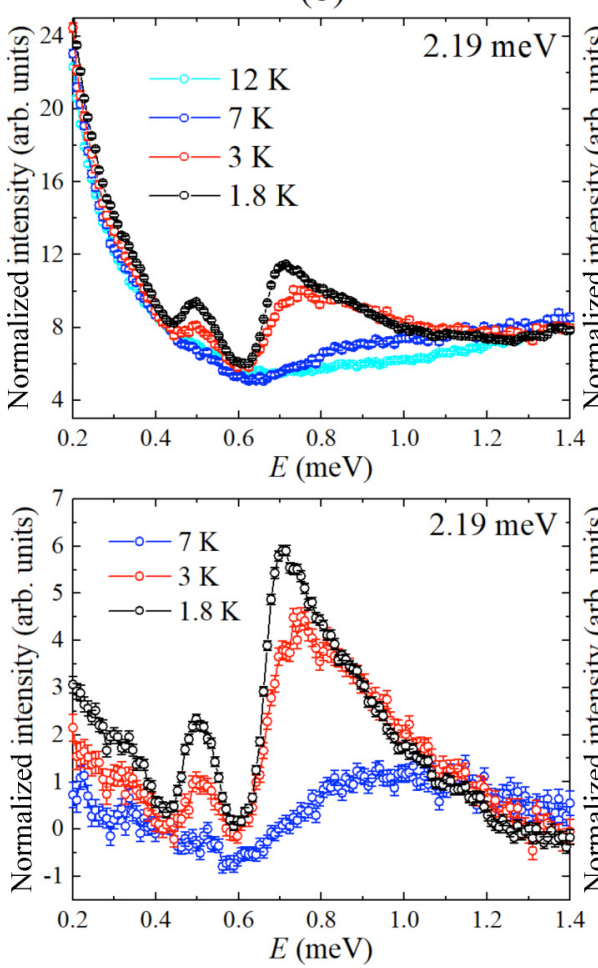

(c)
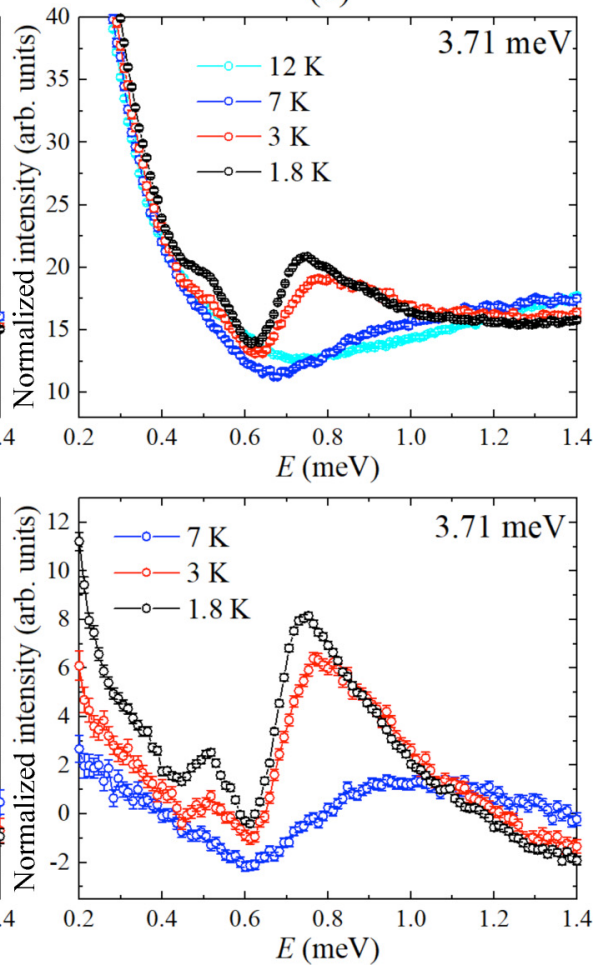

FIG. 11. Energy cuts of INS data, obtained by integrating over the full measured range of $|Q|$, at different incident neutron energies $\left(E_{i}\right)$ showing data before (top) and after (bottom) subtraction of the $12-\mathrm{K}$ data. (a) $E_{i}=1.46 \mathrm{meV}$, (b) $E_{i}=2.19 \mathrm{meV}$, and (c) $E_{i}=3.71 \mathrm{meV}$.

50-gauge high-purity copper wire. When the sample is centered within the coil and the field is pulsed, the voltage induced in the coil is proportional to the rate of change of magnetization with time $(d M / d t)$. The total magnetization is obtained by numerical integration of the signal with respect to time. A subtraction of the integrated signal recorded using an empty coil under the same conditions is used to calculate the magnetization of the sample. The magnetic field is measured via the signal induced within a coaxial ten-turn coil and calibrated using de Haas-van Alphen oscillations within the copper coils of the susceptometer.

\section{Inelastic neutron scattering}

Zero-field inelastic neutron-scattering measurements were performed on a powder sample of $\mathrm{NiI}_{2}(3,5 \text {-lut })_{4}$ (mass $3.5 \mathrm{~g}$ ) using the high-flux chopper setting on the direct geometry time-of-flight spectrometer LET, at the ISIS facility, UK. A primary incident energy $E_{\mathrm{i}}=3.7 \mathrm{meV}$ was used, and repetition rate multiplication provides a number of incident energies with various flux and resolution combinations in the energy window of interest for this compound, of which we focus on the $E_{\mathrm{i}}=2.2 \mathrm{meV}$ data. The sample was mounted in a ${ }^{4} \mathrm{He}$ cryostat and data sets were collected at temperatures of $T=1.7,3,7$, and $12 \mathrm{~K}$, where the latter was treated as a nonmagnetic background. Figure 10 shows the data taken at 12 and $1.8 \mathrm{~K}$ before subtraction.

Figure 11 shows the energy cuts, obtained by integrating over the full measured range of $|Q|$, at different incident neutron energies $E_{i}$. As can be seen, the lines vary only a little with $E_{i}$. At the lowest $E_{i}$ the line shapes of the two features have very similar profiles, with a sharp low-energy onset and slow dropoff at higher energies, as might be expected for a sharp band minimum. The relative size of the two features does not change a great deal with $E_{i}$; specifically the total intensity of the higher energy feature is always larger than that at lower energy. We attribute this difference to the fact that the higher energy mode corresponds to scattering from two degenerate energy bands at zero field, while the lower energy feature arises from just one, as shown in the energy-level diagram of Fig. 8.

\section{Muon spin relaxation}

Zero-field $\mu^{+} \mathrm{SR}$ measurements on $\mathrm{NiI}_{2}(3,5 \text {-lut })_{4}$ were made on the LTF and GPS instruments at the Swiss Muon Source $(\mathrm{S} \mu \mathrm{S})$, Paul Scherrer Institut, Switzerland. Transversefield $\mu^{+}$SR measurements were carried out on a polycrystalline sample using the HAL-9500 spectrometer at $\mathrm{S} \mu \mathrm{S}$. For the zero-field measurements, a powder sample was packed in $\mathrm{Ag}$ foil envelopes (foil thickness $12.5 \mu \mathrm{m}$ ), attached to a silver plate, and mounted on the cold finger of a dilution refrigerator. For transverse-field measurements, a powder sample was packed in foil and glued to a silver holder that was mounted on the cold finger of a dilution refrigerator. Data analysis was carried out using the WIMDA analysis program [88].

In a muon spin-relaxation $\left(\mu^{+} \mathrm{SR}\right)$ experiment, a beam of spin-polarized positive muons is incident upon a material 


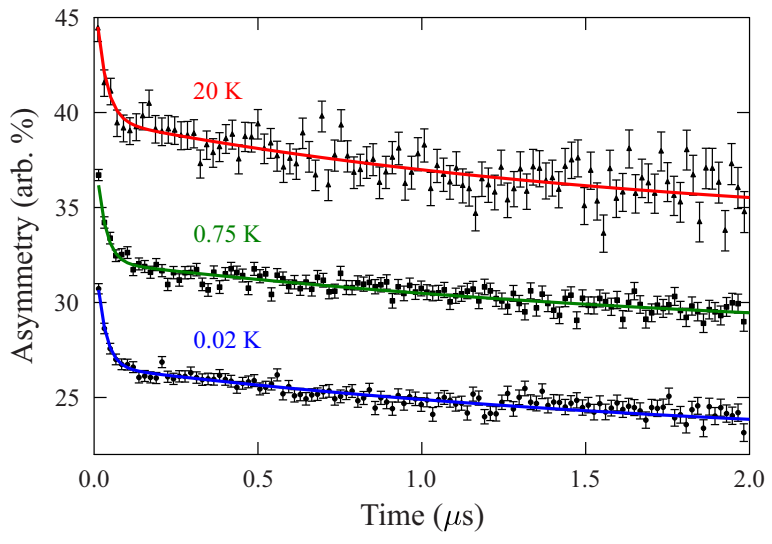

FIG. 12. Asymmetry from ZF $\mu^{+} \mathrm{SR}$ measurements on $\mathrm{NiI}_{2}(3,5-\mathrm{lut})_{4}$ at three different temperatures. (The spectra are offset for clarity.) The solid lines are fits to Eq. (A1).

[59,89]. Muons stop within the sample at particular sites, where their spins precess in the local magnetic field, before decaying after, on average, $2.2 \mu \mathrm{s}$. The observed property of the experiment is the average spin polarization of muon ensemble. In a zero-field (ZF) $\mu^{+} \mathrm{SR}$ experiment the muon spins precess entirely in the internal magnetic field of the sample. If a material shows quasistatic LRO, we expect spontaneous coherent oscillations in $P_{z}(t)$, the longitudinal spin polarization along the initial muon spin direction. In the $\mathrm{TF} \mu^{+} \mathrm{SR}$ experimental geometry [89] the externally applied field $\mu_{0} \boldsymbol{H}_{0}$ is directed perpendicular to the initial muon spin direction. Muons precess about the total magnetic field $\boldsymbol{B}$ at the muon site, which is a vector sum of $\mu_{0} \boldsymbol{H}_{0}$ and fields due to the $\mathrm{Ni}$ (II) moments. The observed property of the experiment is the time evolution of the muon spin polarization $P_{x}(t)$. The Larmor relation between muon precession frequency and local magnetic-field strength $\omega=\gamma_{\mu} B$ (where $\gamma_{\mu} /(2 \pi)=$ $135.5 \mathrm{MHz} \mathrm{T}^{-1}$ is the muon gyromagnetic ratio) allows the determination of the distribution $p(B)$ of local magnetic fields across the sample volume by means of a Fourier transform of $P_{x}(t)$.

Zero-field $\mu^{+} \mathrm{SR}$ measurements. Example ZF spectra spanning the measured temperature range for a powder sample of $\mathrm{NiI}_{2}(3,5 \text {-lut })_{4}$ are shown in Fig. 12. The asymmetry data were fitted in the time domain with two exponentially decaying components and a constant background $A_{\mathrm{bkg}}$,

$$
A(t)=A_{1} e^{-\lambda_{1} t}+A_{2} e^{-\lambda_{2} t}+A_{\mathrm{bkg}},
$$

where $\lambda_{i}$ are relaxation rates. The two separate exponentials capture both fast-relaxing and slow-relaxing components of the data. The fast relaxing component typically arises from muons in bound states [90] whereas the slow relaxing component likely arises from fluctuations of magnetic fields detected by diamagnetic muon states, and is therefore sensitive to the magnetism of the system. The relaxation due to muon bound states is not expected to change with temperature, and as the value of $\lambda_{2} \simeq 38 \mathrm{MHz}$ remained roughly constant over the whole temperature range, it was fixed at this value for the fits. Similarly, the ratio of muons in bound states

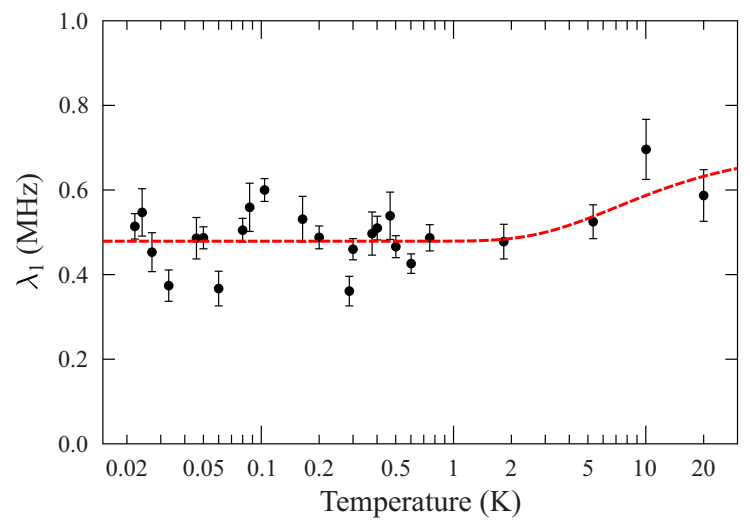

FIG. 13. Values of $\lambda_{1}$ obtained by fitting ZF $\mu^{+} \mathrm{SR}$ data to Eq. (A1). Red dashed line shows $\lambda_{1} \propto \exp \left(-\Delta / k_{\mathrm{B}} T\right)$ with $\Delta=7 \mathrm{~K}$.

to those in diamagnetic states should remain constant with temperature (as the muon site is determined by the structure of the material). Approximately constant values of $A_{1}$ and $A_{2}$ were found over the whole temperature range, as expected, and hence $A_{1}$ and $A_{2}$ were also fixed.

The value of $\lambda_{1}$ as a function of temperature is shown in Fig. 13. At a phase transition, one would expect a dramatic increase in the value of $\lambda_{1}$, indicating that there is a large variation in the values of internal magnetic fields, or a slowing of dynamics. As the value of $\lambda_{1}$ stays approximately constant throughout the whole temperature range this suggests that there is no transition to long-range order even down to the lowest measured temperatures.

Previous work [91] has shown that the relaxation rate $\lambda_{1}$ in a Haldane chain should vary as $\exp \left(-\gamma \Delta / k_{\mathrm{B}} T\right)$, where $\Delta$ is the gap magnitude and the value of the constant $\gamma$ depends on the temperature regime and details of the model. Figure 13 shows this scaling law for $\Delta=7 \mathrm{~K}$ and $\gamma=1$ (expected for low temperatures), suggesting that the slight increase in $\lambda_{1}$ above the gap temperature is consistent with the predicted behavior. Although there is insufficient data density to test which value of $\gamma$ best describes this system, the location of the upturn is supportive of the value $\Delta \approx 7 \mathrm{~K}$ found through other techniques.

There are several other possible causes of the occurrence of the relaxation parametrized by $\lambda_{1}$, all consistent with the realisation of a Haldane phase in this material. One possibility is that chain ends give rise to magnetic moments, which introduce some magnetic-field dynamics that cause the relaxation. Another possibility is that defects in the sample affect the local-field configuration, again leading to a small, fluctuating magnetic field. It is also possible that the muon itself distorts the local environment at the implantation site inducing a small local moment. This has been observed previously in the magnetically disordered state of molecular spin-ladder systems [61], where it was shown that it did not prevent the muon from being a faithful probe of the intrinsic physics of the system.

\section{Electron-spin resonance}

High-field, high-frequency ESR spectra of powdered samples were recorded on a home-built spectrometer at the 
EMR facility, National High Magnetic Field Laboratory, Tallahassee, Florida, USA. Microwave frequencies in the range $52 \leqslant v \leqslant 422 \mathrm{GHz}$ and temperatures $T=3$ and $30 \mathrm{~K}$ were used in the measurement. The instrument is a transmission-type device and uses no resonant cavity. A powdered sample was loaded into a thin Teflon vessel and lowered into a ${ }^{4} \mathrm{He}$ cryostat. The microwaves were generated by a phase-locked Virginia Diodes source, with generating frequency $(13 \pm 1) \mathrm{GHz}$, and equipped with a cascade of frequency multipliers to generate higher harmonic frequencies. The resultant signal was detected using a cold bolometer, and a superconducting magnet generated fields up to $15 \mathrm{~T}$.
[1] S. B. Batten, S. M. Neville, and D. R. Turner, Coordination Polymers: Design, Analysis and Application (Royal Society of Chemistry, Cambridge, UK, 2009).

[2] S. J. Blundell, Contemp. Phys. 48, 275 (2007).

[3] P. A. Goddard, J. L. Manson, J. Singleton, I. Franke, T. Lancaster, A. J. Steele, S. J. Blundell, C. Baines, F. L. Pratt, R. D. McDonald, O. E. Ayala-Valenzuela, J. F. Corbey, H. I. Southerland, P. Sengupta, and J. A. Schlueter, Phys. Rev. Lett. 108, 077208 (2012).

[4] S. Sachdev and B. Keimer, Phys. Today 64(2), 29 (2011).

[5] V. Zapf, M. Jaime, and C. D. Batista, Rev. Mod. Phys. 86, 563 (2014).

[6] P. R. Hammar, M. B. Stone, D. H. Reich, C. Broholm, P. J. Gibson, M. M. Turnbull, C. P. Landee, and M. Oshikawa, Phys. Rev. B 59, 1008 (1999).

[7] T. Hong, Y. H. Kim, C. Hotta, Y. Takano, G. Tremelling, M. M. Turnbull, C. P. Landee, H.-J. Kang, N. B. Christensen, K. Lefmann, K. P. Schmidt, G. S. Uhrig, and C. Broholm, Phys. Rev. Lett. 105, 137207 (2010).

[8] B. C. Watson, V. N. Kotov, M. W. Meisel, D. W. Hall, G. E. Granroth, W. T. Montfrooij, S. E. Nagler, D. A. Jensen, R. Backov, M. A. Petruska, G. E. Fanucci, and D. R. Talham, Phys. Rev. Lett. 86, 5168 (2001).

[9] T. Lancaster, P. A. Goddard, S. J. Blundell, F. R. Foronda, S. Ghannadzadeh, J. S. Möller, P. J. Baker, F. L. Pratt, C. Baines, L. Huang, J. Wosnitza, R. D. McDonald, K. A. Modic, J. Singleton, C. V. Topping, T. A. W. Beale, F. Xiao, J. A. Schlueter, A. M. Barton, R. D. Cabrera et al., Phys. Rev. Lett. 112, 207201 (2014).

[10] V. S. Zapf, D. Zocco, B. R. Hansen, M. Jaime, N. Harrison, C. D. Batista, M. Kenzelmann, C. Niedermayer, A. Lacerda, and A. Paduan-Filho, Phys. Rev. Lett. 96, 077204 (2006).

[11] M. B. Stone, I. A. Zaliznyak, T. Hong, C. L. Broholm, and D. H. Reich, Nature (London) 440, 187 (2006).

[12] F. D. M. Haldane, Phys. Lett. A 93, 464 (1983).

[13] F. D. M. Haldane, Phys. Rev. Lett. 50, 1153 (1983).

[14] I. Affleck, T. Kennedy, E. H. Lieb, and H. Tasaki, Phys. Rev. Lett. 59, 799 (1987).

[15] M. Hagiwara, K. Katsumata, I. Affleck, B. I. Halperin, and J. P. Renard, Phys. Rev. Lett. 65, 3181 (1990).

[16] S. H. Glarum, S. Geschwind, K. M. Lee, M. L. Kaplan, and J. Michel, Phys. Rev. Lett. 67, 1614 (1991).

[17] O. Avenel, J. Xu, J. S. Xia, M.-F. Xu, B. Andraka, T. Lang, P. L. Moyland, W. Ni, P. J. C. Signore, C. M. C. M. van Woerkens, E. D. Adams, G. G. Ihas, M. W. Meisel, S. E. Nagler, N. S. Sullivan, Y. Takano, D. R. Talham, T. Goto, and N. Fujiwara, Phys. Rev. B 46, 8655 (1992).

[18] Y. Uchiyama, Y. Sasago, I. Tsukada, K. Uchinokura, A. Zheludev, T. Hayashi, N. Miura, and P. Böni, Phys. Rev. Lett. 83, 632 (1999).
[19] A. Zheludev, T. Masuda, I. Tsukada, Y. Uchiyama, K. Uchinokura, P. Böni, and S.-H. Lee, Phys. Rev. B 62, 8921 (2000).

[20] T. Sakai and M. Takahashi, Phys. Rev. B 42, 4537 (1990).

[21] K. Wierschem and P. Sengupta, Mod. Phys. Lett. B 28, 1430017 (2014).

[22] K. Wierschem and P. Sengupta, Phys. Rev. Lett. 112, 247203 (2014).

[23] Y.-C. Tzeng, H. Onishi, T. Okubo, and Y.-J. Kao, Phys. Rev. B 96, 060404(R) (2017).

[24] W. J. A. Blackmore et al. (unpublished).

[25] J. Liu, P. A. Goddard, J. Singleton, J. Brambleby, F. Foronda, J. S. Möller, Y. Kohama, S. Ghannadzadeh, A. Ardavan, S. J. Blundell, T. Lancaster, F. Xiao, R. C. Williams, F. L. Pratt, P. J. Baker, K. Wierschem, S. H. Lapidus, K. H. Stone, P. W. Stephens, J. Bendix et al., Inorg. Chem. 55, 3515 (2016).

[26] W. J. A. Blackmore, J. Brambleby, T. Lancaster, S. Clarke, R. D. Johnson, J. Singleton, A. Ozarowski, J. A. Schlueter, Y. Chen, A. M. Arif, S. Lapidus, F. Xiao, R. C. Williams, S. J. Blundell, M. J. Pearce, M. R. Lees, D. Y. Villa, J. A. Villa, J. L. Manson, and P. A. Goddard, New J. Phys. 21, 093025 (2019).

[27] Y. Rahnavard and W. Brenig, Phys. Rev. B 91, 054405 (2015).

[28] S. Ma, C. Broholm, D. H. Reich, B. J. Sternlieb, and R. W. Erwin, Phys. Rev. Lett. 69, 3571 (1992).

[29] I. A. Zaliznyak, S.-H. Lee, and S. V. Petrov, Phys. Rev. Lett. 87, 017202 (2001).

[30] M. Kenzelmann, R. A. Cowley, W. J. L. Buyers, R. Coldea, J. S. Gardner, M. Enderle, D. F. McMorrow, and S. M. Bennington, Phys. Rev. Lett. 87, 017201 (2001).

[31] O. Golinelli, T. Jolicoeur, and R. Lacaze, Phys. Rev. B 45, 9798 (1992).

[32] M. P. Nightingale and H. W. J. Blöte, Phys. Rev. B 33, 659 (1986).

[33] S. R. White and D. A. Huse, Phys. Rev. B 48, 3844 (1993).

[34] O. Golinelli, T. Jolicoeur, and R. Lacaze, Phys. Rev. B 50, 3037 (1994).

[35] S. Todo and K. Kato, Phys. Rev. Lett. 87, 047203 (2001).

[36] S. R. White and I. Affleck, Phys. Rev. B 77, 134437 (2008).

[37] I. Affleck, Phys. Rev. B 46, 9002 (1992).

[38] O. Golinelli, T. Jolicoeur, and R. Lacaze, J. Phys.: Condens. Matter 5, 7847 (1993).

[39] L. P. Regnault, I. A. Zaliznyak, and S. V. Meshkov, J. Phys.: Condens. Matter 5, L677 (1993).

[40] A. M. Tsvelik, Phys. Rev. B 42, 10499 (1990).

[41] A. Abragam and B. Bleaney, Electron Paramagnetic Resonance of Transition Ions (Clarendon, Oxford, 1970).

[42] A. M. Farutin and V. I. Marchenko, J. Exp. Theor. Phys. 104, 751 (2007).

[43] L. P. Regnault, I. Zaliznyak, J. P. Renard, and C. Vettier, Phys. Rev. B 50, 9174 (1994). 
[44] A. Zheludev, Z. Honda, C. L. Broholm, K. Katsumata, S. M. Shapiro, A. Kolezhuk, S. Park, and Y. Qiu, Phys. Rev. B 68 , 134438 (2003).

[45] A. I. Smirnov, V. N. Glazkov, T. Kashiwagi, S. Kimura, M. Hagiwara, K. Kindo, A. Y. Shapiro, and L. N. Demianets, Phys. Rev. B 77, 100401(R) (2008).

[46] A. K. Bera, B. Lake, A. T. M. N. Islam, O. Janson, H. Rosner, A. Schneidewind, J. T. Park, E. Wheeler, and S. Zander, Phys. Rev. B 91, 144414 (2015).

[47] A. K. Bera, B. Lake, A. T. M. N. Islam, and A. Schneidewind, Phys. Rev. B 92, 060412(R) (2015).

[48] J. B. Goodenough, Magnetism and the Chemical Bond (Interscience, New York, 1963).

[49] J. Kanamori, J. Phys. Chem. Solids 10, 87 (1959).

[50] P. W. Anderson, Solid State Phys. 14, 99 (1963).

[51] D. J. Hamm, J. Bordner, and A. F. Schreiner, Inorg. Chim. Acta 7, 637 (1973).

[52] J. M. Law, H. Benner, and R. K. Kremer, J. Phys.: Condens. Matter 25, 065601 (2013).

[53] A. K. Bera, B. Lake, A. T. M. N. Islam, B. Klemke, E. Faulhaber, and J. M. Law, Phys. Rev. B 87, 224423 (2013).

[54] J.-P. Renard, L.-P. Regnault, and M. Verdaguer, in Magnetism: Molecules to Materials (John Wiley \& Sons, Ltd, 2003), Chap. 2, pp. 49-93.

[55] J. C. Bonner and M. E. Fisher, Phys. Rev. 135, A640 (1964).

[56] J. Brambleby, J. L. Manson, P. A. Goddard, M. B. Stone, R. D. Johnson, P. Manuel, J. A. Villa, C. M. Brown, H. Lu, S. Chikara, V. Zapf, S. H. Lapidus, R. Scatena, P. Macchi, Y.-s. Chen, L.-C. Wu, and J. Singleton, Phys. Rev. B 95, 134435 (2017).

[57] H. Mutka, C. Payen, P. Molinié, J. L. Soubeyroux, P. Colombet, and A. D. Taylor, Phys. Rev. Lett. 67, 497 (1991).

[58] A. Zheludev, T. Masuda, K. Uchinokura, and S. E. Nagler, Phys. Rev. B 64, 134415 (2001).

[59] A. Yaouanc and P. Dalmas de Réotier, Muon Spin Rotation, Relaxation and Resonance (Oxford University Press, Oxford, 2004).

[60] B. D. Rainford and G. J. Daniell, Hyperfine Interact. 87, 1129 (1994).

[61] T. Lancaster, F. Xiao, B. M. Huddart, R. C. Williams, F. L. Pratt, S. J. Blundell, S. J. Clark, R. Scheuermann, T. Goko, S. Ward, J. L. Manson, C. Rüegg, and K. W. Krämer, New J. Phys. 20, 103002 (2018).

[62] B. S. Conner, H. D. Zhou, Y. J. Jo, L. Balicas, C. R. Wiebe, J. P. Carlo, Y. J. Uemura, A. A. Aczel, T. J. Williams, and G. M. Luke, Phys. Rev. B 81, 132401 (2010).

[63] E. Čižmár, M. Ozerov, O. Ignatchik, T. P. Papageorgiou, J. Wosnitza, S. A. Zvyagin, J. Krzystek, Z. Zhou, C. P. Landee, B. R. Landry, M. M. Turnbull, and J. L. Wikaira, New J. Phys. 10, 033008 (2008).

[64] N. M. Atherton, Principles of Electron Spin Resonance (Ellis Horwood; PTR Prentice Hall, New York, 1993).
[65] T. Kashiwagi, M. Hagiwara, S. Kimura, Z. Honda, H. Miyazaki, I. Harada, and K. Kindo, Phys. Rev. B 79, 024403 (2009).

[66] A. Zheludev, S. M. Shapiro, Z. Honda, K. Katsumata, B. Grenier, E. Ressouche, L.-P. Regnault, Y. Chen, P. Vorderwisch, H.-J. Mikeska, and A. K. Kolezhuk, Phys. Rev. B 69, 054414 (2004).

[67] M. Hagiwara, Z. Honda, K. Katsumata, A. K. Kolezhuk, and H.-J. Mikeska, Phys. Rev. Lett. 91, 177601 (2003).

[68] G. Xu, J. F. DiTusa, T. Ito, K. Oka, H. Takagi, C. Broholm, and G. Aeppli, Phys. Rev. B 54, 6827(R) (1996).

[69] M. Takigawa, T. Asano, Y. Ajiro, and M. Mekata, Phys. Rev. B 52, R13087 (1995).

[70] T. Sakaguchi, K. Kakurai, T. Yokoo, and J. Akimitsu, J. Phys. Soc. Jpn. 65, 3025 (1996).

[71] A. Zheludev and T. Roscilde, C. R. Phys. 14, 740 (2013).

[72] R. Yu, L. Yin, N. S. Sullivan, J. S. Xia, C. Huan, A. PaduanFilho, N. F. Oliveira Jr, S. Haas, A. Steppke, C. F. Miclea, F. Weickert, R. Movshovich, E.-D. Mun, B. L. Scott, V. S. Zapf, and T. Roscilde, Nature (London) 489, 379 (2012).

[73] T. Hong, A. Zheludev, H. Manaka, and L.-P. Regnault, Phys. Rev. B 81, 060410(R) (2010).

[74] E. S. Sørensen and I. Affleck, Phys. Rev. B 51, 16115 (1995).

[75] X. Wang and S. Mallwitz, Phys. Rev. B 53, R492 (1996).

[76] www.ccdc.cam.ac.uk/structures.

[77] https://wrap.warwick.ac.uk/129863.

[78] P. Goddard et al., The role of zero-point fluctuations in a quantum-disordered spin-dimer compound (STFC ISIS Neutron and Muon Source, 2018), doi: 10.5286/ISIS.E.RB1810129.

[79] SAINT v8.34A (Bruker AXS Inc., Madison, WI, 2013).

[80] SADABS v.2014/2015 (Bruker AXS Inc., Madison, WI, 2014).

[81] SHELXT v.2014/4 (Bruker AXS Inc., Madison, WI, 2014).

[82] SHELXL v.2014/7 (Bruker AXS Inc., Madison, WI, 2014).

[83] International Tables for Crystallography (Wiley, Chichester, 2006), Vol. A, pp. 444-445.

[84] K. V. Kamenev, S. Tancharakorn, N. Robertson, and A. Harrison, Rev. Sci. Instrum. 77, 073905 (2006).

[85] P. Stamenov and J. M. D. Coey, Rev. Sci. Instrum. 77, 015106 (2006).

[86] M. Sawicki, W. Stefanowicz, and A. Ney, Semicond. Sci. Technol. 26, 064006 (2011).

[87] M. J. Coak, C. Liu, D. M. Jarvis, S. Park, M. J. Cliffe, and P. A. Goddard, Rev. Sci. Instrum. (to be published).

[88] F. L. Pratt, Phys. B (Amsterdam, Neth.) 289-290, 710 (2000).

[89] S. J. Blundell, Contemp. Phys. 40, 175 (1999).

[90] T. Lancaster, S. J. Blundell, M. L. Brooks, P. J. Baker, F. L. Pratt, J. L. Manson, C. P. Landee, and C. Baines, Phys. Rev. B 73, 020410(R) (2006).

[91] S. Capponi, M. Dupont, A. W. Sandvik, and P. Sengupta, Phys. Rev. B 100, 094411 (2019). 